\title{
The effect of non-uniform radiative damping on the zonal-mean dynamics of the extratropical middle atmosphere
}

Article

Published Version

Semeniuk, K. and Shepherd, T. G. (2002) The effect of nonuniform radiative damping on the zonal-mean dynamics of the extratropical middle atmosphere. Quarterly Journal of the Royal Meteorological Society, 128 (579). pp. 259-284. ISSN 1477-870X doi: https://doi.org/10.1256/00359000260498888 Available at https://centaur.reading.ac.uk/33047/

It is advisable to refer to the publisher's version if you intend to cite from the work. See Guidance on citing.

Published version at: http://dx.doi.org/10.1256/00359000260498888

To link to this article DOI: http://dx.doi.org/10.1256/00359000260498888

Publisher: Royal Meteorological Society

All outputs in CentAUR are protected by Intellectual Property Rights law, including copyright law. Copyright and IPR is retained by the creators or other copyright holders. Terms and conditions for use of this material are defined in the End User Agreement.

www.reading.ac.uk/centaur 
Central Archive at the University of Reading

Reading's research outputs online 


\title{
The effect of non-uniform radiative damping on the zonal-mean dynamics of the extratropical middle atmosphere
}

\author{
By K. SEMENIUK and T. G. SHEPHERD* \\ University of Toronto, Canada
}

(Received 4 August 2000; revised 30 April 2001)

\begin{abstract}
SUMMARY
The effect of spatial and temporal variations in the radiative damping rate on the response to an imposed forcing or diabatic heating is examined in a zonal-mean model of the middle atmosphere. Attention is restricted to the extratropics, where a linear approach is viable. It is found that regions with weak radiative damping rates are more sensitive in terms of temperature to the remote influence of the diabatic circulation. The delay in the response in such regions can mean that 'downward' control is not achieved on seasonal time-scales. A seasonal variation in the radiative damping rate modulates the evolution of the response and leaves a transient-like signature in the annual mean temperature field.

Several idealized examples are considered, motivated by topical questions. It is found that wave drag outside the polar vortex can significantly affect the temperatures in its interior, so that high-latitude, high-altitude gravitywave drag is not the only mechanism for warming the southern hemisphere polar vortex. Diabatic mass transport through the $100 \mathrm{hPa}$ surface is found to lag the seasonal evolution of the wave drag that drives the transport, and thus cannot be considered to be in the downward control regime. On the other hand, the seasonal variation of the radiative damping rate is found to make only a weak contribution to the annual mean temperature increase that has been observed above the ozone hole.
\end{abstract}

KEYWORDS: Diabatic circulation

\section{INTRODUCTION}

One of the defining properties of the extratropical middle atmosphere is the strongly relaxational nature of diabatic heating. It is this feature, for example, which leads to the zonal-mean 'downward control' behaviour of the diabatic circulation induced by extratropical wave drag (Haynes et al. 1991). The spatio-temporal structure of the zonal wind, temperature and diabatic circulation response to wave drag is sensitive to the radiative damping rate. If the time-scale of the wave drag is much longer than the radiative damping time then the response has a steady-state character; if it is much shorter, then the response has a transient character.

Previous studies of the basic zonal-mean dynamics (e.g. Garcia 1987; Haynes et al. 1991; Holton et al. 1995; Sankey 1998) have used the quasi-geostrophic equations and the Newtonian cooling approximation for the diabatic heating with a constant radiative damping rate. These studies considered the effect of varying the magnitude of the damping rate, but did not address the influence of spatio-temporal variations in the damping rate. Although varying the value of a uniform radiative damping rate gives a sense of the dependence of solutions on this important parameter, there are features that can only be captured with a non-uniform spatial or temporal distribution.

In fact, the radiative transfer characteristics of the middle atmosphere vary significantly in space and time (Andrews et al. 1987). This stems from the pattern of solar heating via the ozone distribution, and from increasing optical thickness with decreasing altitude. The solar heating maximum, and hence the radiative equilibrium temperature maximum, migrates from pole to pole during the annual cycle, and very low radiative equilibrium temperatures are reached in the polar night. Although dynamical heating tends to mitigate some of the more extreme annual temperature variations, especially in

* Corresponding author: Department of Physics, University of Toronto, 60 St George Street, Toronto, Ontario, M5S 1A7, Canada. e-mail: tgs@ atmosp.physics.utoronto.ca

(C) Royal Meteorological Society, 2002. 
the polar night, the underlying radiative equilibrium has, nevertheless, a major impact on the state of the middle atmosphere.

The radiative damping rate is sensitive to the local background temperature: lower background temperatures imply a slower damping rate. As a result, the radiative damping rate has significant latitudinal dependence. In the upper stratosphere the smallest values occur in the winter hemisphere polar regions, while in the lower stratosphere there is an additional minimum in the tropics. The radiative damping rate also varies significantly with altitude due to density stratification. Higher densities at lower altitudes translate into greater optical thickness and weaker damping rates. For a Newtonian cooling approximation to capture these effects in the simplest possible form, the cooling coefficient needs to vary with latitude, altitude and time of year. A parametrization of the cooling coefficient in terms of temperature would make the system nonlinear and complicate the analysis. However, the quasi-geostrophic, Newtonian-cooling formulation of the dynamics used in previous studies suffices if the problem is treated as a perturbation to a climatological basic state.

In this paper, the impact of spatial variations of the radiative damping rate on the dynamics will be considered in the context of several problems. The central element in these problems is local deviation from downward control due to weak radiative damping rates. Beyond the spatial variations, temporal variations in the radiative damping rate can affect the interpretation of annual mean temperature anomalies and the behaviour of transients that are present through interannual variability.

Numerical experiments indicate that gravity-wave drag can have a significant remote influence on the stratospheric southern hemisphere high latitudes during the winter season (Garcia and Boville 1994). Because of the weaker planetary-wave drag in the southern hemisphere, gravity-wave drag plays a relatively more important role than in the northern hemisphere in producing dynamical heating. The downward control principle would suggest that high-latitude wave drag in the mesosphere makes the largest contribution, and the very weak radiative damping rates that prevail at these latitudes translate into a large impact in terms of the temperature. However, with a weak radiative damping rate the concentration of streamlines induced by high-latitude mesospheric wave drag below the forcing level would take longer than a season to occur. This means that the effect of wave drag in the middle and lower stratosphere, which in the southern hemisphere winter is believed to be confined outside the polar vortex, cannot be ignored. Even though the radiative damping rate is large enough in midlatitudes for downward control to be achieved on a seasonal time-scale, the weak damping rate within the vortex could prevent the expulsion of diabatic circulation streamlines. Therefore, it is useful to investigate the manner in which the spatial distribution of radiative damping in the polar night affects the influence of adjacent regions of wave drag.

Calculation of the seasonal cycle of mass in the lowermost stratosphere (Appenzeller et al. 1996) shows a lag of the mass outflow into the troposphere compared to the peak wave drag above $100 \mathrm{hPa}$ (about $16 \mathrm{~km}$ ). The mass flux through the $100 \mathrm{hPa}$ surface is an important part of the analysis. The weak radiative damping at these altitudes means that downward control of the diabatic circulation by wave drag is not attained, and the pattern of mass flux will reflect this. It is important to distinguish this effect from seasonal variations of the mass of the lowermost stratosphere.

The radiative damping rate is also a key factor affecting transients associated with interannual variations in forcing (e.g. planetary wave drag or ozone heating) in the middle atmosphere. At high latitudes and in the lower stratosphere, transients will last longer due to the smaller radiative damping rate. As is evident from the results to be 
presented here, transients can persist longer than a year and can, therefore, obscure the interpretation of annual mean diagnostics. For example, the annual mean temperature increase observed above the region of seasonal ozone loss in the southern hemisphere polar regions (e.g. Fig. 1 of Ramaswamy et al. 1996) could, perhaps, be partly explained by transience. This is in addition to warming attributable to wave-drag redistribution or additional infrared heating from the troposphere (through the cold, ozone-depleted region).

The annual behaviour of transients such as those associated with the formation of the ozone hole will also be affected by the seasonal variation of the radiative damping rate. The time dependence of the radiative damping rate is greatest in the polar regions owing to the extreme swing in temperatures between the winter solstice (polar night) and summer solstice. In contrast, the variations in tropical temperature and radiative damping rate are smaller, as the sun passes over the equator twice a year and the region is always sunlit. The strong temporal variation in the damping rate in the polar regions allows the possibility of a net temperature change even if the net radiative heating change vanishes. It is interesting to quantify the importance of this effect.

In this paper we examine the effect of the spatio-temporal dependence of the radiative damping rate on the zonal-mean dynamics pertaining to the issues described above. There is no way to cover all of parameter space, so the results presented are merely illustrative of the possibilities. Thus, our conclusions are largely qualitative. In section 2 the model and methodology are presented. The effects of spatial and temporal variations in the radiative damping rate on the cases of 'switch-on' and steady periodic forcing are considered in section 3. In section 4 the annual mean signature of temporal variations in the radiative damping rate is examined. The paper concludes with a discussion in section 5 .

\section{Model AND METHODOLOGY}

The zonal-mean dynamics of the middle atmosphere can be reasonably modelled using the TEM (Transformed Eulerian Mean) equations (Andrews et al. 1987). The Coriolis term typically dominates the nonlinear terms in the zonal momentum equation in the extratropics, and the system is close to being in thermal-wind balance. Thus for this study, the quasi-geostrophic (linear) version of the TEM equations is employed:

$$
\begin{gathered}
\frac{\partial \bar{u}}{\partial t}-f \bar{v}^{*}=\mathcal{F}, \\
\frac{\partial \bar{T}}{\partial t}+S \bar{w}^{*}=\mathcal{Q}, \\
\frac{1}{a \cos \phi} \frac{\partial\left(\bar{v}^{*} \cos \phi\right)}{\partial \phi}+\frac{1}{\rho} \frac{\partial\left(\rho \bar{w}^{*}\right)}{\partial z}=0, \\
f \frac{\partial \bar{u}}{\partial z}=-\frac{R}{a H} \frac{\partial \bar{T}}{\partial \phi} .
\end{gathered}
$$

Here $t$ is time, $\bar{u}$ is the zonal-mean zonal wind and $\bar{T}$ is the zonal-mean temperature; $\bar{v}^{*}$ and $\bar{w}^{*}$ are the residual meridional and vertical velocities, respectively; $a$ is the radius of the earth, $\rho$ is the density, $S$ is the static stability, and $f=2 \Omega \sin \phi$ is the Coriolis parameter; $\phi$ is latitude, $z$ is a log-pressure vertical coordinate, $R$ is the gas constant for dry air, and $H$ is the density-scale height; $\mathcal{F}$ represents the EP (Eliassen-Palm) flux divergence (or wave drag) and $\mathcal{Q}$ the diabatic heating. 
The diabatic heating consists of two parts: a solar heating $\mathcal{Q}_{\mathrm{s}}$ and a temperature dependent long-wave cooling $\mathcal{Q}_{1}(\bar{T})$. A reasonable approximation is New tonian cooling $\mathcal{Q} \equiv \mathcal{Q}_{1}(\bar{T})+\mathcal{Q}_{\mathrm{S}}=-\alpha\left(\bar{T}-\bar{T}_{\text {rad }}\right)$ where $\bar{T}_{\text {rad }}$ is the prescribed radiative equilibrium temperature, and $\alpha$ depends on $\phi, z$ and $t$. Highly idealized profiles of the radiative damping rate $\alpha$ (Fig. 1) are inferred from a comprehensive radiative transfer scheme (Fomichev and Blanchet 1995) applied to the COSPAR International Reference Atmosphere climatology. The largest variations in space and time occur at high latitudes where the system moves in and out of polar night with the march of the seasons. The radiative damping rate also varies significantly with altitude due to the exponential increase in density with decreasing altitude.

The dependence of $\alpha$ on $\bar{T}$ is not allowed for in this formulation, which makes the system separable into $\mathcal{F}$-driven and $\mathcal{Q}_{\mathrm{s}}$-driven parts. The latter can be further subdivided into effects due to $\bar{T}_{\text {rad }}$ and to a hypothetical perturbation $\delta \bar{T}_{\text {rad }}$ (e.g. ozone loss). An imposed diabatic forcing is equivalent to a change in $\bar{T}_{\text {rad }}$. Defining the wave drag component $\bar{u}_{\mathrm{F}}, \bar{T}_{\mathrm{F}}, \bar{v}_{\mathrm{F}}^{*}$ and $\bar{w}_{\mathrm{F}}^{*}$ by

$$
\begin{gathered}
\frac{\partial \bar{u}_{\mathrm{F}}}{\partial t}-f \bar{v}_{\mathrm{F}}^{*}=\mathcal{F} \\
\frac{\partial \bar{T}_{\mathrm{F}}}{\partial t}+S \bar{w}_{\mathrm{F}}^{*}=-\alpha\left(\bar{T}_{\mathrm{F}}\right),
\end{gathered}
$$

the diabatic forcing perturbation component $\bar{u}_{\mathrm{Q}}, \bar{T}_{\mathrm{Q}}, \bar{v}_{\mathrm{Q}}^{*}$ and $\bar{w}_{\mathrm{Q}}^{*}$ by

$$
\begin{gathered}
\frac{\partial \bar{u}_{\mathrm{Q}}}{\partial t}-f \bar{v}_{\mathrm{Q}}^{*}=0 \\
\frac{\partial \bar{T}_{\mathrm{Q}}}{\partial t}+S \bar{w}_{\mathrm{Q}}^{*}=-\alpha\left(\bar{T}_{\mathrm{Q}}-\delta \bar{T}_{\mathrm{rad}}\right),
\end{gathered}
$$

and the radiative equilibrium component $\bar{u}_{\mathrm{R}}, \bar{T}_{\mathrm{R}}, \bar{v}_{\mathrm{R}}^{*}$ and $\bar{w}_{\mathrm{R}}^{*}$ by

$$
\begin{gathered}
\frac{\partial \bar{u}_{\mathrm{R}}}{\partial t}-f \bar{v}_{\mathrm{R}}^{*}=0 \\
\frac{\partial \bar{T}_{\mathrm{R}}}{\partial t}+S \bar{w}_{\mathrm{R}}^{*}=-\alpha\left(\bar{T}_{\mathrm{R}}-\bar{T}_{\mathrm{rad}}\right),
\end{gathered}
$$

the combined fields satisfy the full equations:

$$
\begin{gathered}
\frac{\partial\left(\bar{u}_{\mathrm{F}}+\bar{u}_{\mathrm{Q}}+\bar{u}_{\mathrm{R}}\right)}{\partial t}-f\left(\bar{v}_{\mathrm{F}}^{*}+\bar{v}_{\mathrm{Q}}^{*}+\bar{v}_{\mathrm{R}}^{*}\right)=\mathcal{F} \\
\frac{\partial\left(\bar{T}_{\mathrm{F}}+\bar{T}_{\mathrm{Q}}+\bar{T}_{\mathrm{R}}\right)}{\partial t}+S\left(\bar{w}_{\mathrm{F}}^{*}+\bar{w}_{\mathrm{Q}}^{*}+\bar{w}_{\mathrm{R}}^{*}\right)=-\alpha\left\{\bar{T}_{\mathrm{F}}+\bar{T}_{\mathrm{Q}}+\bar{T}_{\mathrm{R}}-\left(\bar{T}_{\mathrm{rad}}+\delta \bar{T}_{\mathrm{rad}}\right)\right\} .
\end{gathered}
$$

We are interested in the first two components of the solution. The forcing, $\mathcal{F}$, can be some idealized distribution, based on general circulation model (GCM) fields, or based on observed climatology.

This model does not include a number of important elements. One is advective nonlinearity, which prevents the separation of solutions into non-interacting mechanically and radiatively forced components. This is justified by the relative weakness of the advection terms in the extratropics. Another neglected effect is nonlinearity in the 


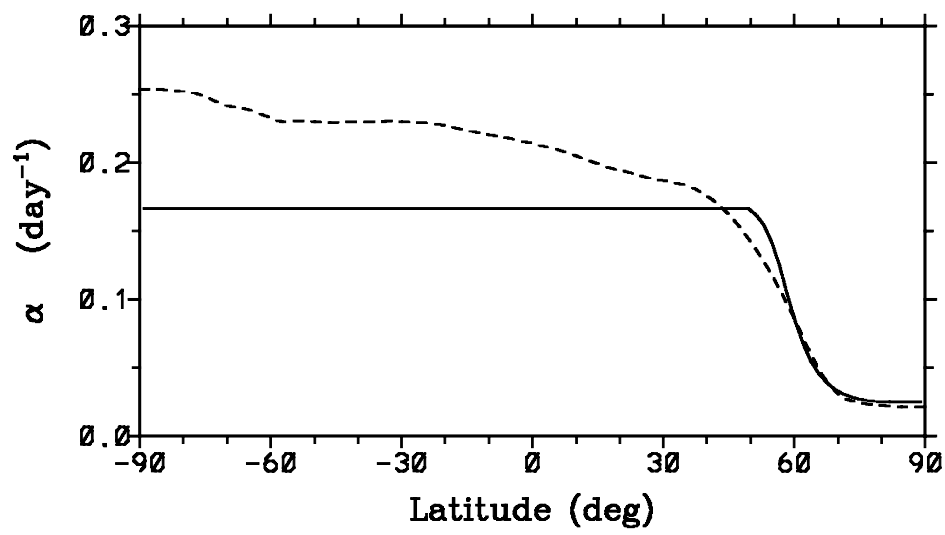

(a)

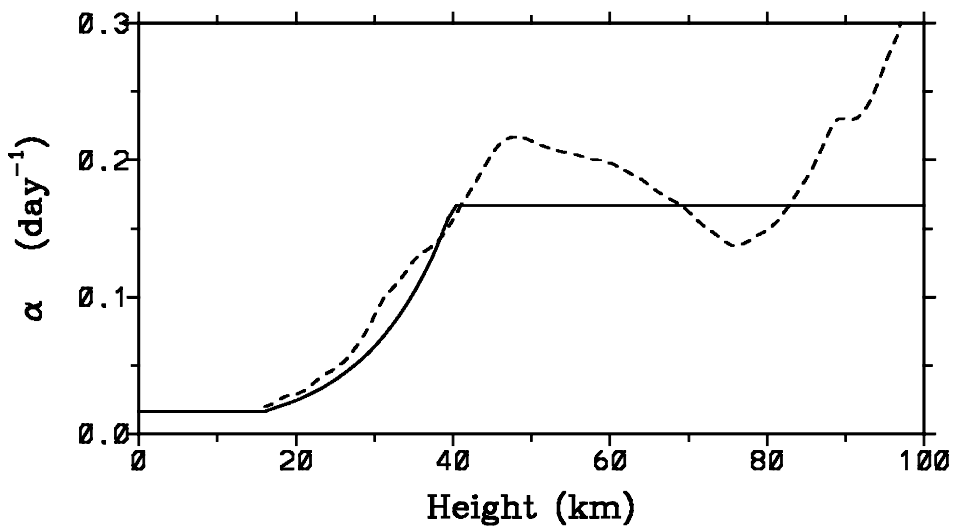

(b)

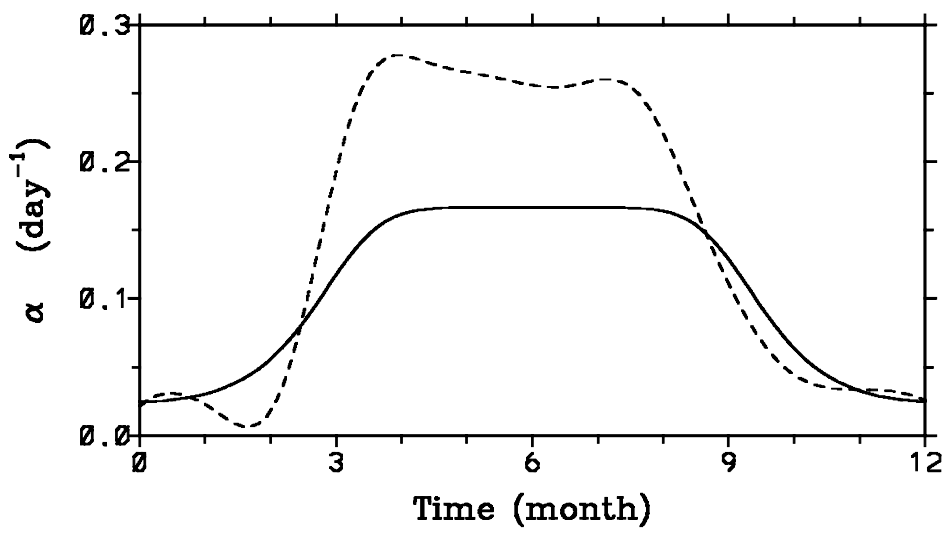

(c)

Figure 1. Profiles of damping rates (a) $\alpha(\phi)$, (b) $\alpha(z)$, and (c) $\alpha\left(90^{\circ}, t\right)$ in units of day ${ }^{-1}$. Idealized profiles (solid lines) are based on sections of $\alpha \equiv\left\{Q\left(\bar{T}_{\mathrm{rad}}\right)-\mathcal{Q}\left(\bar{T}_{\mathrm{rad}}+50 \mathrm{~K}\right)\right\} / 50 \mathrm{~K}$ derived from a realistic radiative transfer scheme (dashed curves): (a) corresponds to a section in latitude at $z=50 \mathrm{~km}$ in January, (b) to a section in height at $\phi=10^{\circ}$ in January, and (c) to a section in time at $\phi=90^{\circ}$ and $z=50 \mathrm{~km}$. (Idealized profiles are arbitrarily limited to $(1 / 6)$ day $^{-1}$ in regions where the damping rate is higher, as the region of interest is the stratosphere.) See text for further details. 
diabatic heating and wave drag. With a comprehensive radiative transfer scheme for the diabatic heating, if the system is to be kept in the climatological regime with the right temperature range and $\alpha$ distribution then a realistic $\mathcal{F}$ field would be required. The wave drag would also respond to changes in the zonal wind and cannot really be regarded as fixed. Nevertheless, useful insight into the basic dynamics can be obtained without including such complex effects. Thus, we treat the wave drag as fixed and use the Newtonian cooling approximation for the diabatic heating in a linear model framework.

Following previous studies (e.g. Garcia 1987; Haynes et al. 1991) we consider the response of the system with variable $\alpha$ to switch-on and steady periodic forcing with a simple distribution. This allows a straightforward comparison with existing work. Although an idealized forcing is used, it is sufficient for the basic analysis undertaken here.

Using the separability property of the system, we set $\bar{T}_{\text {rad }}$ to zero in all the experiments. Three separate idealized cases of $\alpha=\alpha(\phi), \alpha=\alpha(\phi, t)$ and $\alpha=\alpha(z)$ are considered, as noted earlier. Variation in $\alpha$ introduces new complexities to the asymptotic analysis of Haynes et al. (1991) so a numerical analysis is performed instead. Asymptotic analysis may be possible in the $\alpha=\alpha(z)$ case.

The $\alpha(\phi)$ profile (Fig. 1(a)) is motivated by the large latitudinal variation of $\alpha$ at high latitudes in the winter hemisphere at the transition to polar night. This case is intended to analyse the influence of wave drag outside the polar vortex on the interior of the vortex. The seasonal variation in $\alpha$ is also greatest at high latitudes due to the transition to polar night. The chosen form of $\alpha(\phi, t)$ uses the distribution given in Fig. 1(a) during winter solstice:

$$
\frac{1}{\alpha(\phi, t)}=\frac{1}{16}\left(\frac{1}{\alpha(\phi)}-6 \text { days }\right)\left\{1+\cos \left(\frac{2 \pi t}{365}\right)\right\}^{4}+6 \text { days. }
$$

The time profile of $\alpha(\phi, t)$ is given in Fig. 1(c) (for $\phi=90^{\circ}$ ). This approximation does not capture the phase shift with latitude seen in the realistic radiative damping rate, and gives a weaker annual mean damping rate at high latitudes.

The effect of variation of $\alpha$ with altitude is treated in the $\alpha(z)$ case (Fig. 1(b)) and is relevant for the problem of the influence of mesospheric gravity-wave drag on the stratosphere and for the problem of the time dependence of mass flux through the $16 \mathrm{~km}$ (100 hPa) surface.

The experiments use an idealized forcing of the form $\mathscr{F}=F_{0} Y_{\mathrm{F}}(\phi) Z_{\mathrm{F}}(z) H(t)$ where the time-dependent part, $H(t)$, is either a step function or $\cos (\omega t)$ and where

$$
Y_{\mathrm{F}}(\phi)= \begin{cases}\frac{1}{2}+\frac{1}{2} \cos \left[\frac{2 \pi}{\phi_{2}-\phi_{1}}\left\{\phi-\frac{1}{2}\left(\phi_{1}+\phi_{2}\right)\right\}\right] & \text { for } \phi_{1} \leq \phi \leq \phi_{2} \\ 0 & \text { otherwise }\end{cases}
$$

and

$$
Z_{\mathrm{F}}(z)= \begin{cases}\frac{1}{2}+\frac{1}{2} \cos \left[\frac{2 \pi}{z_{2}-z_{1}}\left\{z-\frac{1}{2}\left(z_{1}+z_{2}\right)\right\}\right] & \text { for } z_{1} \leq z \leq z_{2} \\ 0 & \text { otherwise, }\end{cases}
$$

with $F_{0}=-5 \mathrm{~m} \mathrm{~s}^{-1} \mathrm{day}^{-1}$. The position and latitudinal scale of the forcing is varied depending on the profile of $\alpha$ being investigated. 
The idealized diabatic heating perturbation used in experiments dealing with the ozone hole is given by $\Delta \mathcal{Q}=Q_{0} Y(\phi) Z(z) H(t)$, with $Q_{0}=-0.35 \mathrm{~K} \mathrm{day}^{-1}$ and where

$$
Y(\phi)= \begin{cases}\left\{\frac{1}{2}+\frac{1}{2} \cos \left(\pi \frac{\phi_{2}-\phi}{\phi_{2}-\phi_{1}}\right)\right\}^{1 / 2} & \text { for } \phi_{1} \leq \phi \leq \phi_{2} \\ 0 & \text { otherwise }\end{cases}
$$

with $\phi_{1}=65^{\circ}$ and $\phi_{2}=90^{\circ}$,

$$
Z(z)= \begin{cases}\frac{1}{2}+\frac{1}{2} \cos \left[\frac{2 \pi}{z_{2}-z_{1}}\left\{z-\frac{1}{2}\left(z_{1}+z_{2}\right)\right\}\right] & \text { for } z_{1} \leq z \leq z_{2} \\ 0 & \text { otherwise }\end{cases}
$$

with $z_{1}=10 \mathrm{~km}$ and $z_{2}=26 \mathrm{~km}$, and

$$
H(t)= \begin{cases}\left(t_{2}-t\right) /\left(t_{2}-t_{1}\right) & \text { for } t_{1} \leq t \leq t_{2} \\ \frac{1}{2}+\frac{1}{2} \cos \left[\frac{2 \pi}{t_{3}-t_{2}}\left\{t-\frac{1}{2}\left(t_{2}+t_{3}\right)\right\}\right] & \text { for } t_{2} \leq t \leq t_{3} \\ 0 & \text { otherwise, }\end{cases}
$$

with $t_{1}=1.5, t_{2}=4$ and $t_{3}=5.5$ months.

\section{RESPONSE TO SWITCH-ON AND PERIODIC FORCING WITH SPATIALLY AND TEMPORALLY VARIABLE $\alpha$}

The switch-on forcing regime corresponds to infra-seasonal time-scales when, for example, the wintertime surf zone develops and persists until the spring equinox season. As a point of reference we take the uniform-damping case which has been studied by Haynes et al. (1991). The response of the system to a forcing with a step-function time dependence has been investigated in some detail by them with asymptotic and numerical solutions (their section 3(c) and Fig. 6, respectively). The response evolves towards a steady state which has a downward control character. The steady-state limit is not reached by the atmosphere but is approached closely during the winter solstice seasons (Rosenlof and Holton 1993).

Some aspects of steady state with spatially variable $\alpha$ can be inferred a priori. In steady state, $\bar{w}^{*}$ is independent of $\alpha$ and is determined by the latitudinal distribution of streamlines which are all vertically aligned below the region of $\mathcal{F}$ (this follows from the quasi-geostrophic downward control formula of Haynes et al. 1991). So from the steady version of (2) with $\bar{T}_{\text {rad }} \equiv 0$, which is $S \bar{w}^{*}=-\alpha \bar{T}$, it can be seen that the distribution of $\bar{T}$ will vary depending on $\alpha$. This is in addition to the variation imposed by $\bar{w}^{*}$ which is zero above the forcing region and decays inversely to the density below the forcing region. For $\alpha$ varying only in latitude (Fig. 1(a)) this requires that $\mathcal{F}$ overlap the high latitude regions for there to be a steady-state effect. If this is the case then there will be an amplified $\bar{T}$ response in the region overlapping the smaller values of $\alpha$. The effect can be quite large at the altitudes of the forcing. For $\alpha$ varying only in altitude (Fig. 1(b)) there will be an increased $\bar{T}$ response in steady state at lower altitudes. The increase in $\bar{T}$ will be small if the wave drag is over a scale height above the weak $\alpha$ region. If $\alpha^{-1}$ increases proportionally to the density below the forcing region, however, then the $\bar{T}$ 
response can be uniform in $z$. However, the rate of evolution to steady state is roughly proportional to $\alpha$, and this situation will not develop in the course of a season.

The forcing used for the following experiments has the distribution described in section 2. In the switch-on forcing case the system is marched in time for 120 model days after the forcing is turned on. The switch-on forcing case can only give insight into part of the response to time-dependent forcing. Thus, we also consider the timeharmonic behaviour of the system. Middle-atmosphere wave drag has a cyclical time dependence following the annual cycle. Mesospheric gravity-wave drag has opposite signs in the two solstice seasons and a roughly zero annual mean. Planetary-wave drag in the stratosphere occurs primarily during the winter season and is single-signed, so it has a non-zero annual mean component which is in steady state assuming linearity and no interannual variability. In addition to annually varying forcing, higher harmonics corresponding to half a year and a quarter of a year are considered.

\section{(a) The case where $\alpha$ is uniform}

The periodic forcing case for the problem when $\alpha$ is uniform has been investigated in a number of studies (e.g. Garcia 1987; Haynes et al. 1991; Holton et al. 1995; Sankey 1998). The spatio-temporal structure of the solutions is sensitive to the value of the ratio $\omega / \alpha$, where $\omega$ is the frequency of the forcing. The adiabatic or transient limit corresponds to $\omega / \alpha \rightarrow \infty$ and the steady-state limit to $\omega / \alpha \rightarrow 0$. This is demonstrated by a scaling analysis in Holton et al. (1995), reproduced below, which also applies for the intermediate time regime.

The system (1)-(4) with the Newtonian cooling approximation can be rearranged into a single equation for $\bar{w}^{*}$. Assuming that the time dependence is harmonic, with constant frequency $\omega$, the substitutions $\mathcal{F}=\operatorname{Re}\left(F \mathrm{e}^{\mathrm{i} \omega t}\right), \mathcal{Q}_{s}=\operatorname{Re}\left(Q \mathrm{e}^{\mathrm{i} \omega t}\right)$ and $\bar{w}^{*}=$ $\operatorname{Re}\left(w \mathrm{e}^{\mathrm{i} \omega t}\right)$ give this equation the form

$$
\begin{aligned}
& \frac{\partial}{\partial z}\left(\frac{1}{\rho} \frac{\partial(\rho w)}{\partial z}\right)+\left(\frac{\mathrm{i} \omega}{\mathrm{i} \omega+\alpha}\right) \frac{N^{2}}{4 \Omega^{2} a^{2} \cos \phi} \frac{\partial}{\partial \phi}\left(\frac{\cos \phi}{\sin ^{2} \phi} \frac{\partial w}{\partial \phi}\right) \\
& \quad=\left(\frac{\mathrm{i} \omega}{\mathrm{i} \omega+\alpha}\right) \frac{R / H}{4 \Omega^{2} a^{2} \cos \phi} \frac{\partial}{\partial \phi}\left(\frac{\cos \phi}{\sin ^{2} \phi} \frac{\partial Q}{\partial \phi}\right)+\frac{1}{2 \Omega a \cos \phi} \frac{\partial}{\partial \phi}\left(\frac{\cos \phi}{\sin \phi} \frac{\partial F}{\partial z}\right) .
\end{aligned}
$$

The solution to this equation consists of a linear superposition of a component due to solar heating, the first term on the right-hand side, and a component due to the imposed force, the second term on the right-hand side. We are interested at the moment in the solution pertaining to the imposed force, so the first term on the right-hand side can be excluded. The adiabatic limit corresponds to the limit $\mathrm{i} \omega /(\mathrm{i} \omega+\alpha) \rightarrow 1$. In this case, scaling analysis of (19), taking $a \Delta \phi$ as the horizontal scale and $\Delta z$ as the vertical scale of $\rho w$, yields

$$
\Delta z \sim \max \left\{\frac{2 \Omega \sin \phi}{N} a \Delta \phi, \frac{4 \Omega^{2} \sin ^{2} \phi}{N^{2}} \frac{(a \Delta \phi)^{2}}{H}\right\}
$$

outside the forcing region, where the terms on the left-hand side of (19) balance. The first value on the right-hand side of (20) corresponds to $\Delta z<H$, and the second value corresponds to $\Delta z>H$. So for shallow forcing the ratio of the vertical scale to the meridional scale is the ratio of the Coriolis frequency to the buoyancy frequency. For deep forcing, which is the relevant middle-atmospheric case, the vertical scale is much larger, varying as the square of the meridional scale. 
In the intermediate case when $\alpha>\omega$, scaling analysis gives

$$
\Delta z \sim \max \left\{\left(\frac{\alpha}{\omega}\right)^{1 / 2} \frac{2 \Omega \sin \phi}{N} a \Delta \phi,\left(\frac{\alpha}{\omega}\right) \frac{4 \Omega^{2} \sin ^{2} \phi}{N^{2}} \frac{(a \Delta \phi)^{2}}{H}\right\}
$$

outside the forcing region. The additional factors involving $\alpha / \omega$ imply that the vertical scale of the solution is greater for a given meridional scale than in the adiabatic regime. Specifically, the coefficient of the second term on the left-hand side of (19) gets smaller as $\alpha / \omega$ increases, which requires the first term on the left-hand side to decrease also in order to maintain balance. As a result the vertical variation of the solution decreases, or equivalently its vertical scale increases. In the steady-state limit $\alpha / \omega \rightarrow \infty$, the second term on the left-hand side of (19) disappears; then $\rho w$ has no vertical variation outside the forcing region and is zero outside the latitude band containing the force (i.e. downward control).

The solutions have a component in phase with the forcing, and a component $\pi / 2$ out of phase (Sankey 1998), which arises from the induced diabatic heating. The outof-phase component reflects the finite radiative damping time-scale in the system. This simple time dependencefollows from the fact that the balance zonal-mean system has no homogeneous modes and can have no evolution without forcing. In the limit $\alpha / \omega \rightarrow \infty$ the component of the solution which is out of phase with the forcing diminishes to zero.

The temporal behaviour was considered in some detail by Sankey (1998). The relative lag of $\bar{w}^{*}$ and $\bar{T}$ is given by $\tan ^{-1}(\omega / \alpha)$ at all points in the meridional plane. The lag between the upwelling and the temperature, therefore, increases for decreasing $\alpha$ and decreases for increasing $\alpha$. Since this is a pointwise result, the relation will still apply if $\alpha$ is spatially variable. However, the result is based on the time-harmonic decomposition of the governing equations so it will not apply if $\alpha$ is time dependent, in which case there is mixing of harmonics.

The start-up transients produced by steady oscillatory forcing behave much like those for switch-on forcing considered by Haynes et al. (1991). Based on the difference between the solutions for the first two periods and those in the long time limit (not shown), the decay rate is found to have no dependence on $\omega$. Even for the fast damping rate used here of $(1 / 6)$ day $^{-1}$ the transients persisted longer than a year. Reducing the radiative damping rate to $(1 / 40)$ day $^{-1}$ significantly extended the duration of the transients. The longevity of the start-up transients has implications for annual periodic forcing such as the ozone hole, which is located in the polar late-winter/spring lower stratosphere where the radiative damping rate is weak. We consider this issue in more detail in section 4 .

\section{(b) The $\alpha(\phi)$ case}

The stream function and temperature response induced by switch-on forcing are shown in Figs. 2 and 3 at a couple of times during the progression of the system to steady state. The $\alpha(\phi)$ used is given in Fig. 1(a). If the forcing sits completely outside the region of weak radiative damping, then the system evolves to steady state at such a rate as if this region did not exist. Fields that penetrate into this region do exhibit some differences in the transient regime. Streamlines tend to reach to higher latitudes and the induced temperature is stronger (not shown). These effects are persistent if there is some overlap of the forcing with the region of weaker $\alpha$, as is the case for Figs. 2(a) and (c), and Figs. 3(a) and (c). Compared to the uniform damping case the differences in the mass stream function are small. The temperature, however, exhibits a much larger amplitude in the region of small $\alpha$. The temperature maximum of the warming region below the 
(a)

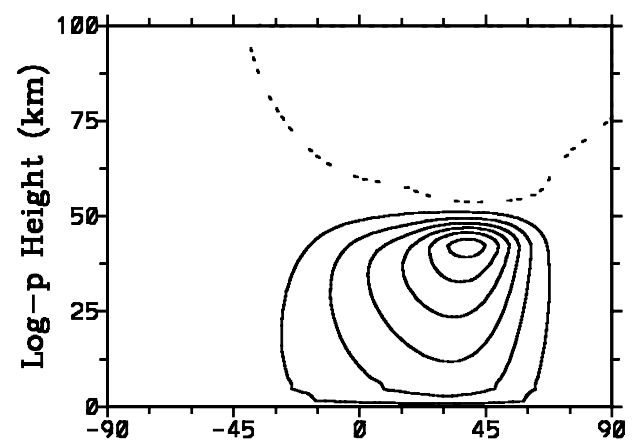

(c)

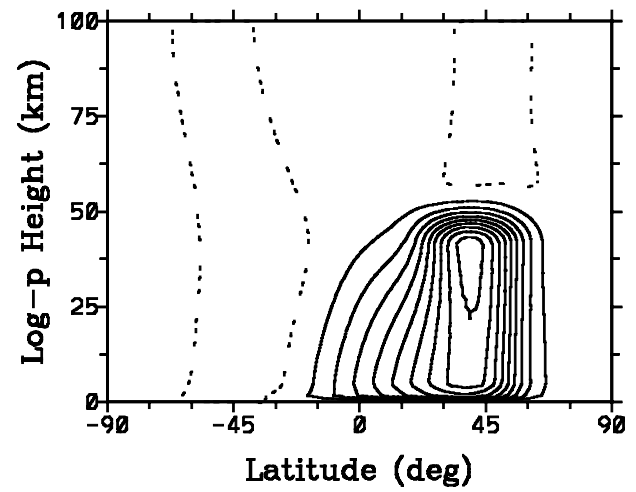

(b)

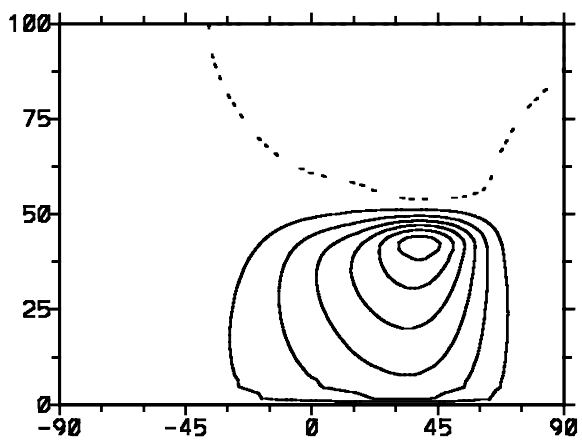

(d)

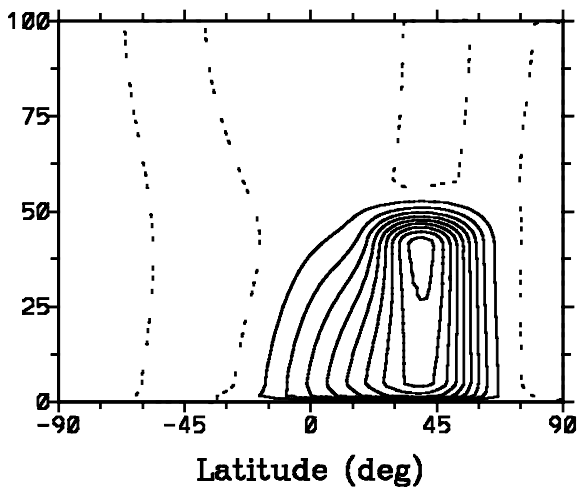

Figure 2. Contour plot of the stream function: (a) and (b) at 10 days, (c) and (d) at 95 days for switch-on forcing overlapping the region of small damping rate; (a) and (c) are for the $\alpha(\phi)$ case, (b) and (d) correspond to a uniform $\alpha=(1 / 6)$ day $^{-1}$. The force distribution parameters are $\phi_{1}=15^{\circ}, \phi_{2}=75^{\circ}, z_{1}=40 \mathrm{~km}$ and $z_{2}=60 \mathrm{~km}$. All contour intervals are $0.5 \mathrm{~kg} \mathrm{~m}^{-1} \mathrm{~s}^{-1}$; intermittent-dotted contours are zero. See text for further details.

forcing and nearest the pole moves to higher latitudes and is nearly doubled in magnitude. In regions of smaller $\alpha$ the circulation is able to drive $\bar{T}$ further from radiative equilibrium. The larger temperature response persists in steady state, as discussed earlier.

Next we consider the response to annually periodic forcing. Since $\alpha$ changes significantly during the annual cycle at high latitudes, we replace the $\alpha$ profile given in Fig. 1(a) with an annual mean of the $\alpha(\phi, t)$ profile. The annual mean profile has a much larger damping rate at high latitudes. Figures 4 and 5 show the $\cos (\omega t)$ component of the stream function and temperature for forcing at two different latitudes. Noticeable differences in the stream function occur only if the streamlines overlap the region of small $\alpha$ at high latitudes, which is the case in Figs. 4 and 5, panels (c) and (d), but not in (a) and (b). The equilibrated oscillatory state at high latitudes has a weaker $\bar{w}^{*}$ but a stronger temperature response which is nearly double that of the uniform $\alpha$ case. There is also more spreading of the circulation into very high latitudes, which contributes to the increase in temperature inside the weak $\alpha$ region.

\section{(c) The $\alpha(\phi, t)$ case}

We now consider the effect of an annual variation in $\alpha$, which is most prominent at high latitudes. Periodic forcing with annual, semi-annual and three-month periods was 
(a)

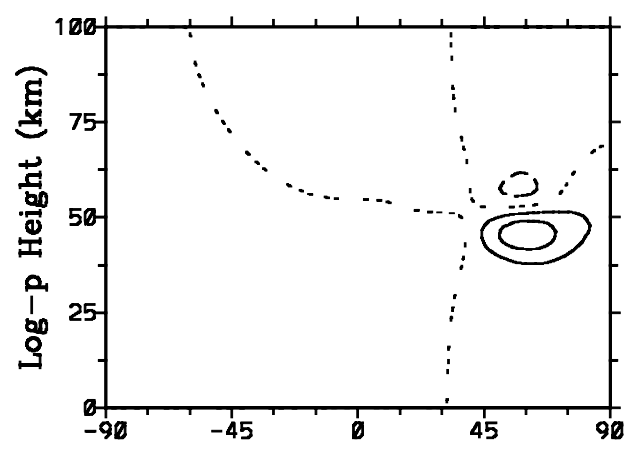

(c)

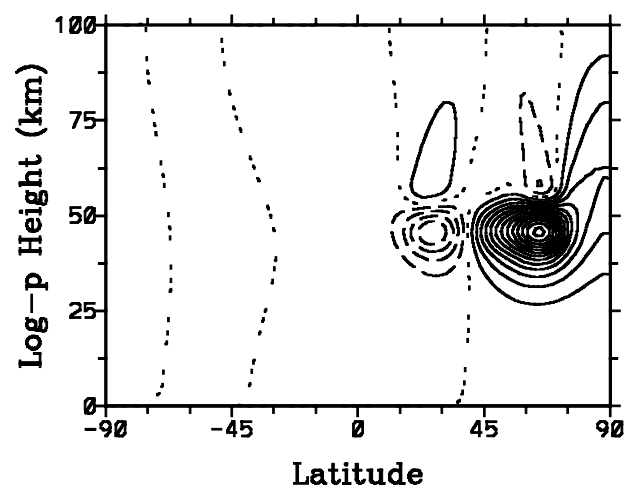

(b)

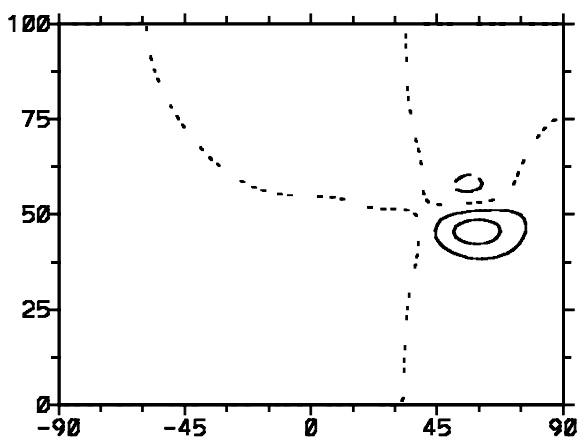

(d)

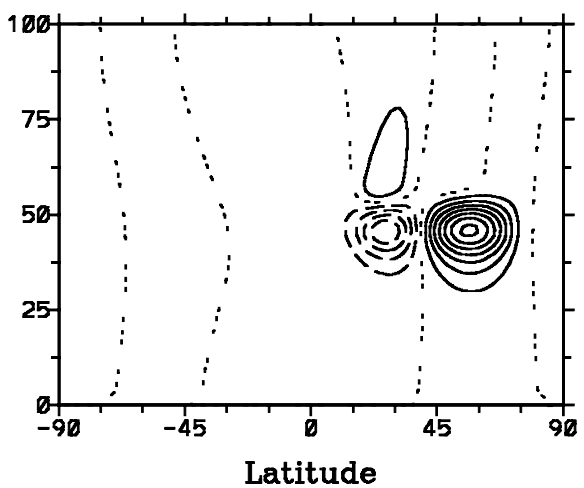

Figure 3. As Fig. 2 but for temperature. All contour intervals are $1 \mathrm{~K}$; dashed contours are negative.

applied to the system. Consideration of the $\cos (\omega t)$ solution component (not shown) indicates that the higher-frequency harmonic is least affected by the annual periodicity of the radiative damping rate. From Fig. 6 it is evident that $\alpha(t)$ is imposing an annual modulation of the response but is not deforming the sinusoidal form of the solution for the higher-frequency forcing (Fig. 6(b)) as much as for the lower-frequency forcing (Fig. 6(a)). The reason for this is that higher-frequency forcing experiences either the low or high values of $\alpha$ during each period as if $\alpha$ were uniform. This leads primarily to a modulation of the solution amplitude in the form of the time dependence of $\alpha$ (Fig. 6(b)). At the other extreme, lower-frequency forcing that has periods longer than a year (not considered here) experiences an average $\alpha$ value and hence is less sensitive to its time dependence. For forcing with a frequency close to that of the radiative damping rate the impact on the time dependence of the solutions is greatest. This can be seen in Fig. 6(a) for the 'climatological' annually periodic solution, where the response during the time of small $\alpha$ is strongly deformed from a sinusoidal form.

A comparison of $\bar{w}^{*}$ for annually averaged and time-dependent $\alpha(\phi, t)$, at a point on the $16 \mathrm{~km}$ surface at high latitudes, is given in Fig. 7. Peak downwelling occurs almost 1.5 months later in the $\alpha(\phi, t)$ case for annually periodic forcing (Fig. 7(a)). As $\omega$ increases, the phase difference between the annually averaged and time-dependent $\alpha$ cases decreases. The annually varying radiative damping rate leads to a more transient distribution in the stream function (not shown) since the system spends part of the time with damping rates much weaker than in the annual mean damping case. 
(a)

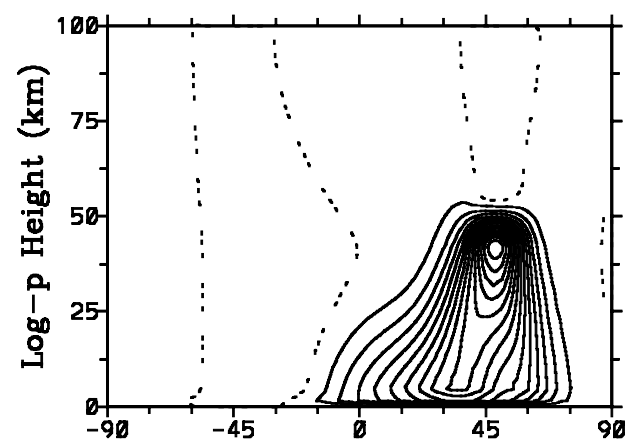

(c)

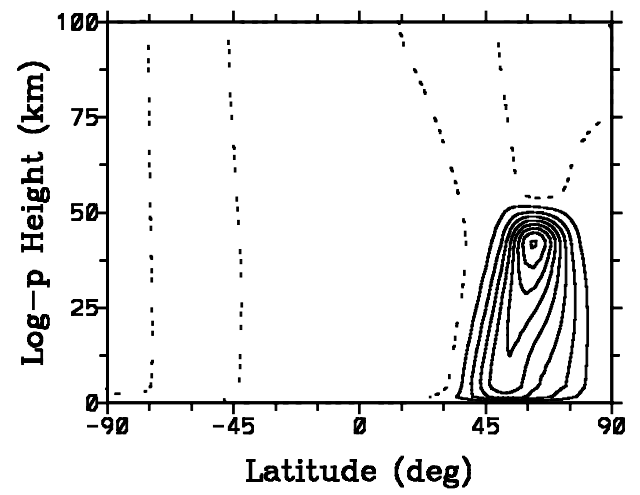

(b)

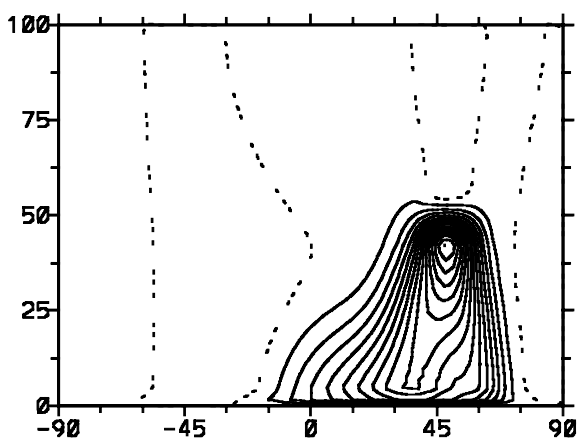

(d)

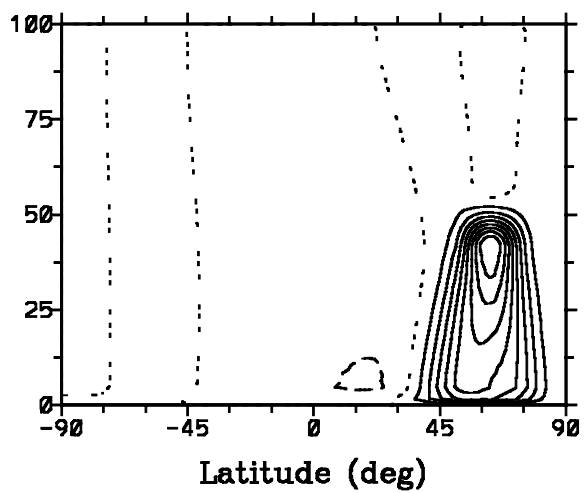

Figure 4. Contour plots of the $\cos (\omega t)$ component of the stream function produced by annually periodic forcing: (a) and (c) show solutions with $\alpha(\phi)$ set to the annual mean of $\alpha(\phi, t)$, with lower values of $\alpha$ over the polar regions; (b) and (d) correspond to a uniform $\alpha=(1 / 6)$ day $^{-1}$. The force distribution parameters are $\phi_{1}=35^{\circ}$ for (a) and (b), $\phi_{1}=50^{\circ}$ for (c) and (d), $\phi_{2}=\phi_{1}+30^{\circ}, z_{1}=40 \mathrm{~km}$ and $z_{2}=60 \mathrm{~km}$. All contour intervals are $0.2 \mathrm{~kg} \mathrm{~m}^{-1} \mathrm{~s}^{-1}$; intermittent-dotted contours are zero, dashed contours are negative. See text for further details.

\section{(d) The $\alpha(z)$ case}

Figures 8 and 9 present snapshots of the evolution of the system to steady state in response to switch-on forcing. The model $\alpha$ is set to the profile in Fig. 1(b). It is apparent that the transience persists for longer periods at lower levels. In Fig. 8(c) and (d) it can be seen that at the forcing levels, where the damping rate is the same, the stream function has a nearly identical distribution in the two cases. Where the damping rates are longer the stream function is still in an earlier stage in its evolution. In particular, it is less confined in latitude and streamlines take a longer time to concentrate directly below the forcing. The effect on the stream function is more noticeable than in the $\alpha(\phi)$ case because of the asymmetric nature of the circulation response, with streamlines penetrating downward instead of sideways. Even though the circulation is weaker at lower altitudes, the temperature response is greater than in the uniform $\alpha$ case (Fig. 9). As noted above this is due to the weaker radiative damping at these levels.

Reducing the latitudinal scale of the forcing reduces the degree of temperature penetration downward at a given time, since the transience is increased; the maximal penetration of solutions below the forcing level occurs in steady state. When $\alpha$ is uniform, the temperature decays exponentially below the forcing level in steady state with the same scale height as the density, and does not depend on the latitudinal scale of 
(a)

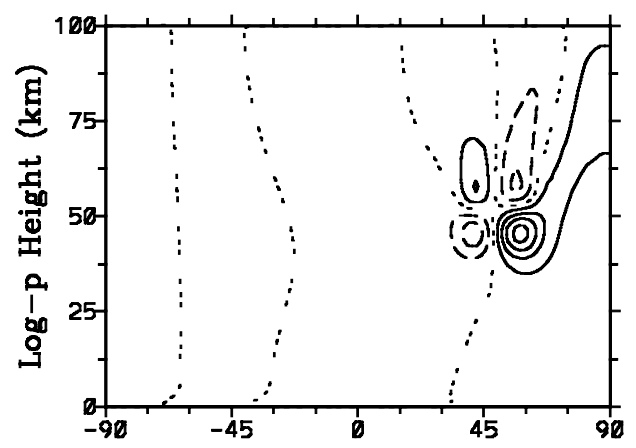

(c)

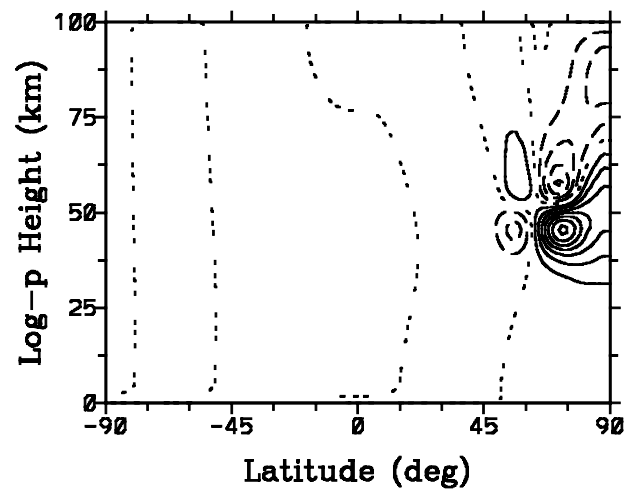

(b)

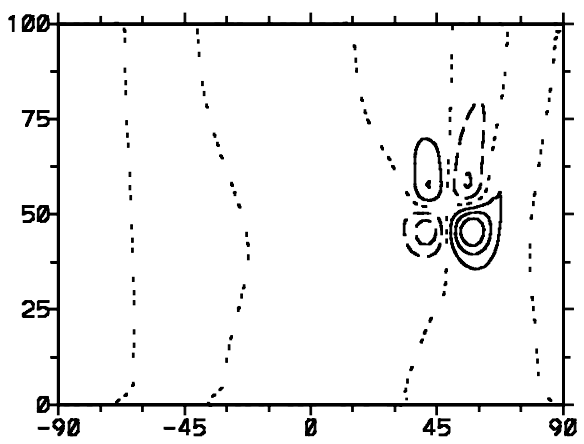

(d)

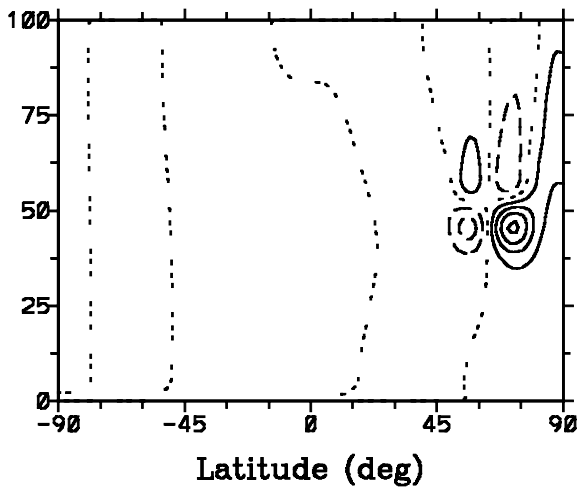

Figure 5. As Fig. 4 but for temperature. All contour intervals are $2 \mathrm{~K}$.

the forcing. As noted previously, for the $\alpha(z)$ case the temperature falls off more slowly below the forcing level. There is an indication of this in Fig. 9(c), where the temperature response decays in $z$ at about half the rate of the uniform $\alpha$ case.

The forcing distribution in the real atmosphere (or GCMs) extends through all levels of the midlatitude stratosphere. To see the behaviour of forcing at lower levels we repeat the experiment above but with the forcing at a lower level. On the $16 \mathrm{~km}$ surface, where $\alpha(z)=(1 / 60) \mathrm{day}^{-1}$, the system takes longer than a season to approach steady state (Fig. 10). The damping rate is sufficiently slow for the use of forcing of larger horizontal scale not to result in a significant change in the rate of evolution to steady state.

The results for the periodic-forcing case, with $\alpha$ varying with altitude below the forcing region, are presented in Figs. 11 and 12. As in the steady-forcing case, the effect on mass streamline behaviour is significant because streamlines penetrate downward into the region of weak $\alpha$ regardless of the latitude or altitude of the forcing. In this region the system appears to be trapped in a transient state (Figs. 11(c) and (d)). Such a combination of transience with a weak damping rate leads to a stronger temperature response even near the pole (Fig. 12(c)). Once again, the diminished concentration of streamlines in latitude translates into a weaker $\bar{w}^{*}$ but one that extends further poleward (and equatorward).

The behaviour of $\bar{w}^{*}$ on the $16 \mathrm{~km}$ surface compared to the uniform $\alpha$ case is given in Fig. 13. The dependence of the phase lag on $\alpha$ is most apparent for the high $\omega$ case 

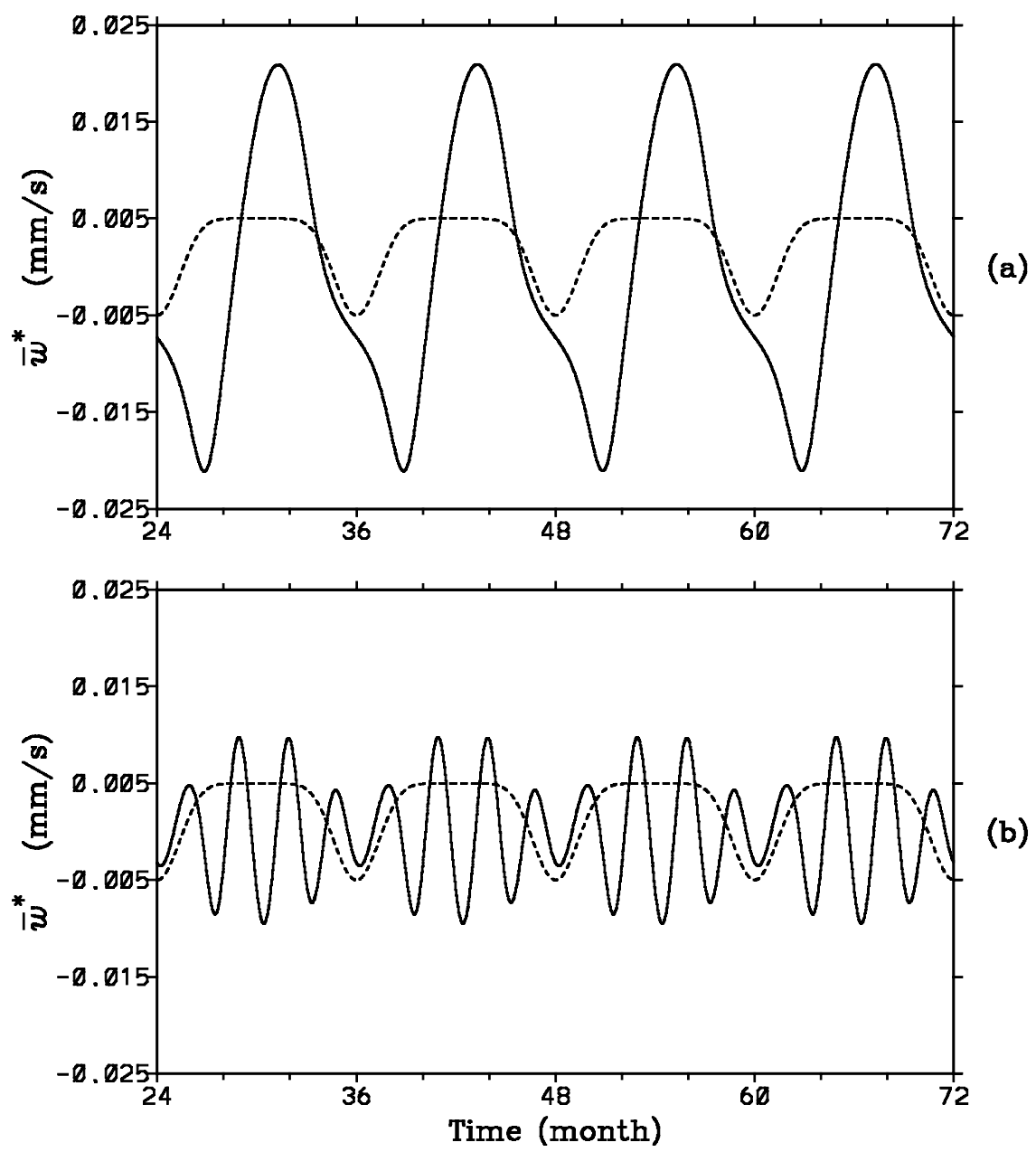

(b)

Figure 6. Time series of $\bar{w}^{*}$ on the $16 \mathrm{~km}$ surface at $76^{\circ}$ for $\alpha(\phi, t)$ (solid lines). The time dependence of $\alpha$ (dashed lines) is represented with an arbitrary amplitude: (a) is for $\omega$ corresponding to a period of 1 year, and (b) for a period of (1/4) year. The force distribution parameters are $\phi_{1}=50^{\circ}, \phi_{2}=80^{\circ}, z_{1}=40 \mathrm{~km}$ and $z_{2}=60 \mathrm{~km}$. See text for further details.

(Fig. 13(b)) where there is a small (about 0.2 of a month) shift in the peaks of the $\alpha(z)$ (solid curve) and uniform $\alpha=(1 / 6)$ day $^{-1}$ (long-dashed curve) responses. This shift is relatively smaller for the low $\omega$ case (Fig. 13(a)), which is consistent with the system having a steady-state behaviour in the limit $\omega \rightarrow 0$. The maximum vertical velocity leads the forcing by a longer time interval for the low $\omega$ case, reaching about 3.5 months in the annually periodic forcing case (the peak downwelling occurs about 2.5 months after the peak positive forcing).

It would appear that vertical variation in the radiative damping rate does not have a significant effect on the lag between the peak of the forcing and the upwelling. As an additional test, the altitude of the annually periodic forcing was reduced by $20 \mathrm{~km}$ (not shown). The effect at $16 \mathrm{~km}$ was a decrease in the lag between the downwelling and forcing to about 1.5 months and the practical disappearance of the phase difference between the uniform and vertically varying $\alpha$ cases. The largest difference between the 


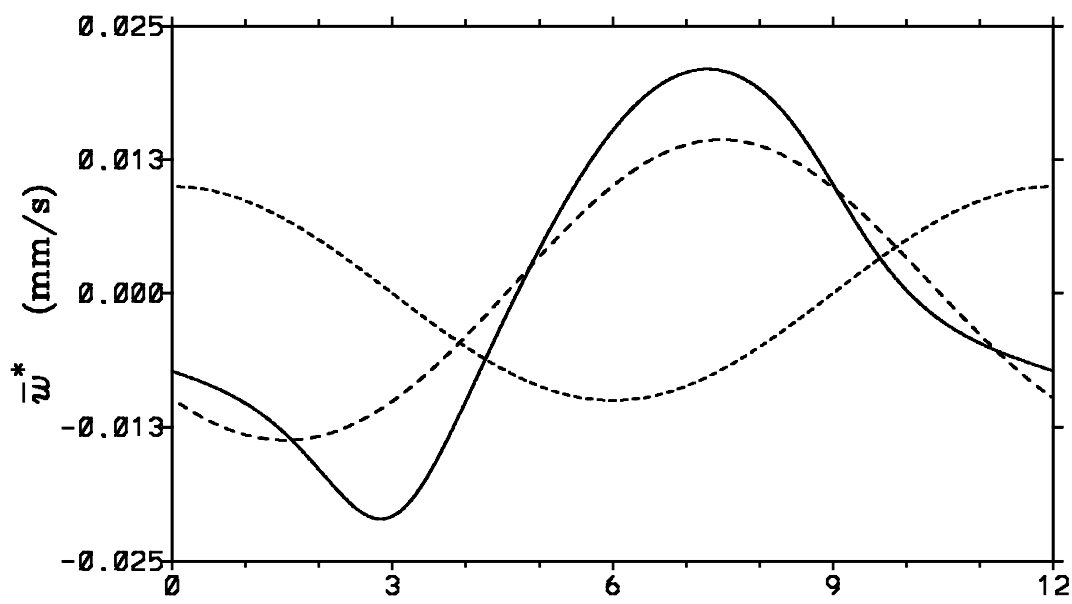

(a)

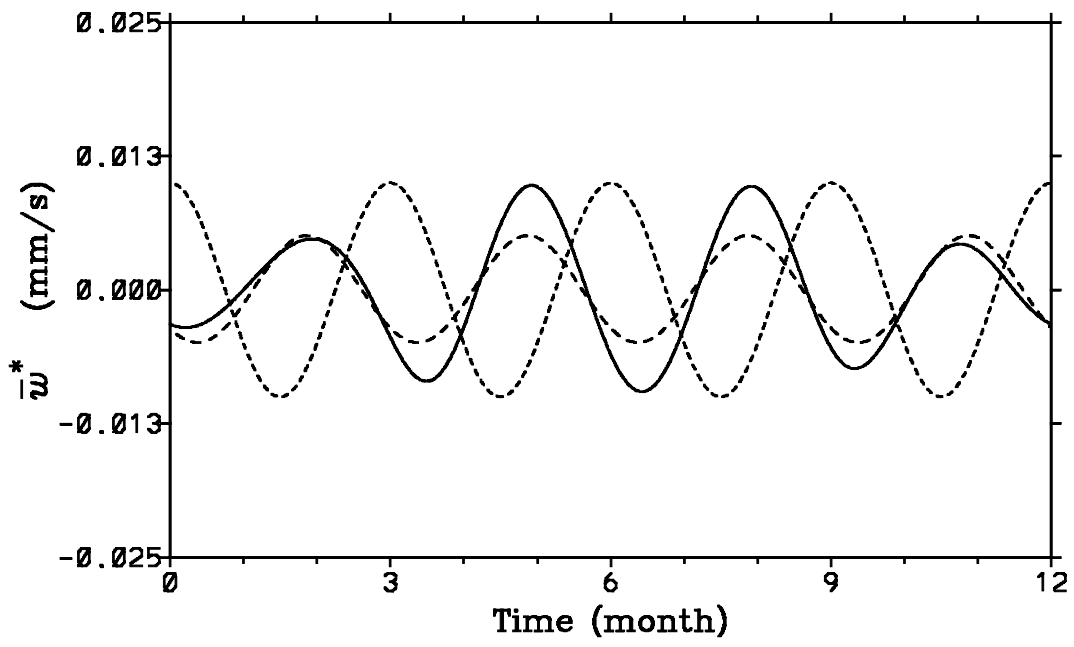

(b)

Figure 7. Time series of $\bar{w}^{*}$ on the $16 \mathrm{~km}$ surface at $76^{\circ}$ for uniform $\alpha(\phi)$ (long-dashed line) and for $\alpha(\phi, t)$ (solid line) for $\omega$ corresponding to: (a) a period of 1 year, and (b) a period of (1/4) year. The time-dependence of the forcing (short-dashed line) is represented with an arbitrary amplitude. The force distribution is the same as in Fig. 6. See text for further details.

two cases was in amplitude: the small radiative damping rate at $16 \mathrm{~km}$ in the $\alpha(z)$ case resulting in a much weaker $\bar{w}^{*}$.

\section{ANNUAL MEAN SignatURE OF $\alpha(t)$}

A property of the quasi-geostrophic equations is that under conditions of no interannual variability and time-independent $\alpha$, their annual mean form is the same as their steady-state form:

$$
\begin{gathered}
-f\left\langle\bar{v}^{*}\right\rangle=\langle\mathcal{F}\rangle \\
S\left\langle\bar{w}^{*}\right\rangle=-\alpha\left\langle\left(\bar{T}-\bar{T}_{\mathrm{rad}}\right)\right\rangle \equiv-\alpha\langle\Delta \bar{T}\rangle .
\end{gathered}
$$


(a)

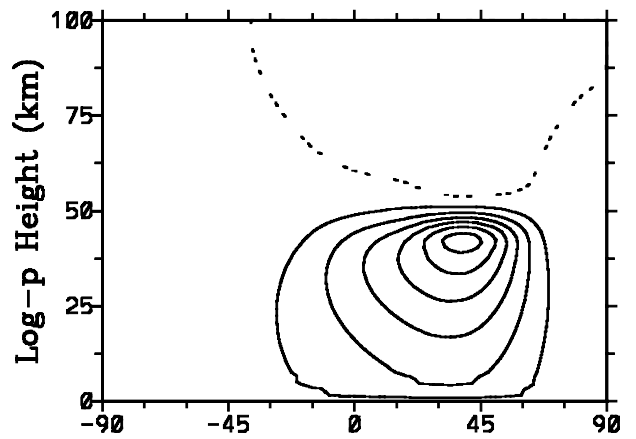

(c)

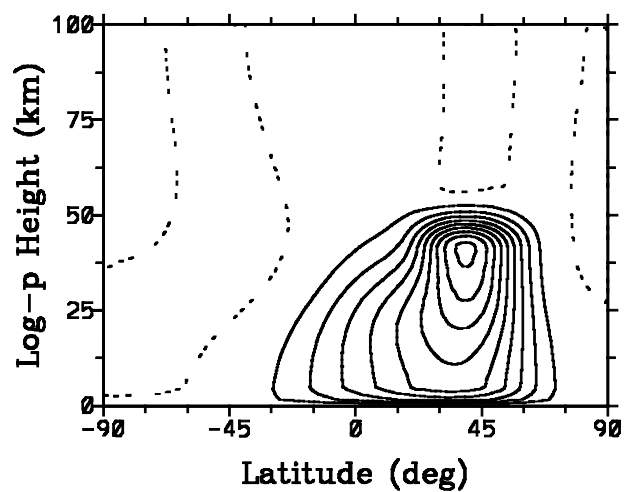

(b)

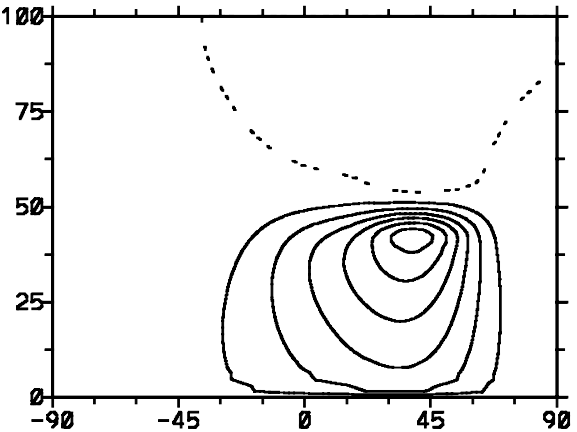

(d)

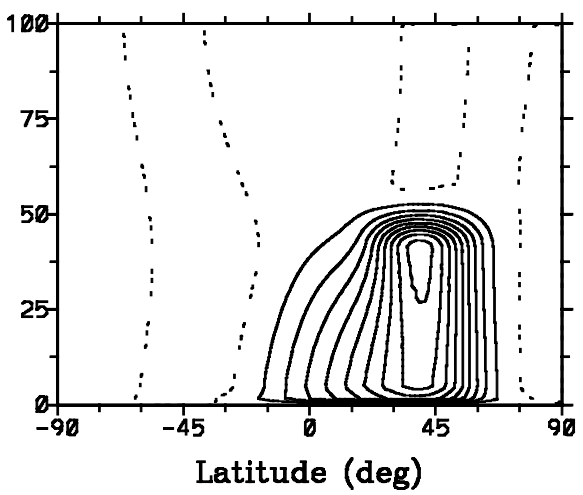

Figure 8. Contour plots of the stream function: (a) and (b) at 10 days, (c) and (d) at 95 days for switch-on forcing at $50 \mathrm{~km}$; (a) and (c) are for the $\alpha(z)$ case, (b) and (d) correspond to a uniform $\alpha=(1 / 6)$ day $^{-1}$. The force distribution parameters are $\phi_{1}=15^{\circ}, \phi_{2}=75^{\circ}, z_{1}=40 \mathrm{~km}$ and $z_{2}=60 \mathrm{~km}$. All contour intervals are $0.5 \mathrm{~kg} \mathrm{~m}^{-1} \mathrm{~s}^{-1}$; intermittent-dotted contours are zero. See text for further details.

This gives rise to the constraint of annual mean downward control. Time dependence in $\alpha$ introduces additional annual mean correlation terms in the quasi-geostrophic annualmean thermodynamic equation

$$
S\left\langle\bar{w}^{*}\right\rangle=-\langle\alpha\rangle\left\langle\left(\bar{T}-\bar{T}_{\text {rad }}\right)\right\rangle-\left\langle\alpha^{\prime}\left(\bar{T}^{\prime}-\bar{T}_{\text {rad }}^{\prime}\right)\right\rangle
$$

where $\alpha^{\prime}=\alpha-\langle\alpha\rangle$ and $\bar{T}^{\prime}=\bar{T}-\langle\bar{T}\rangle$. The annual mean zonal wind equation has no additional terms and annual mean downward control still holds when $\alpha$ is a function of time (as long as $\left\langle\bar{u}_{t}\right\rangle=0$ ).

The significance of the correlation terms in the thermodynamic equation is that the temperature will have a different annual mean distribution than the steady-state one. In the case of an imposed diabatic forcing alone (e.g. the ozone hole case), which can be treated as a modification of the radiative equilibrium temperature, $\delta \bar{T}_{\text {rad }}$, we have

$$
\langle\alpha \Delta \bar{T}\rangle=\left\langle\alpha \delta \bar{T}_{\mathrm{rad}}\right\rangle
$$

since $\left\langle\bar{w}^{*}\right\rangle=0$ everywhere. Hence,

$$
\langle\alpha\rangle\left\langle\Delta \bar{T}-\delta \bar{T}_{\mathrm{rad}}\right\rangle=-\left\langle\alpha^{\prime}\left(\Delta \bar{T}-\delta \bar{T}_{\mathrm{rad}}\right)^{\prime}\right\rangle .
$$


(a)

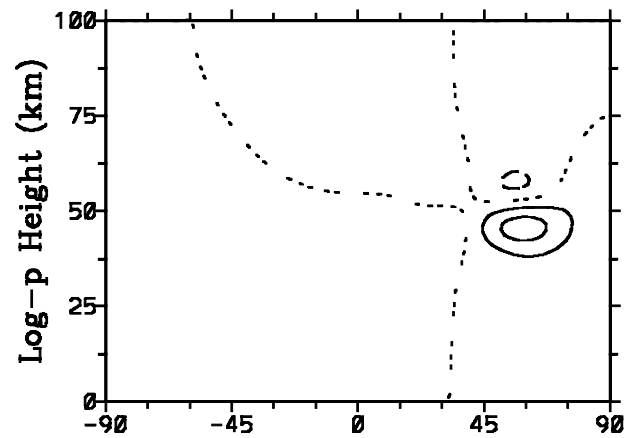

(c)

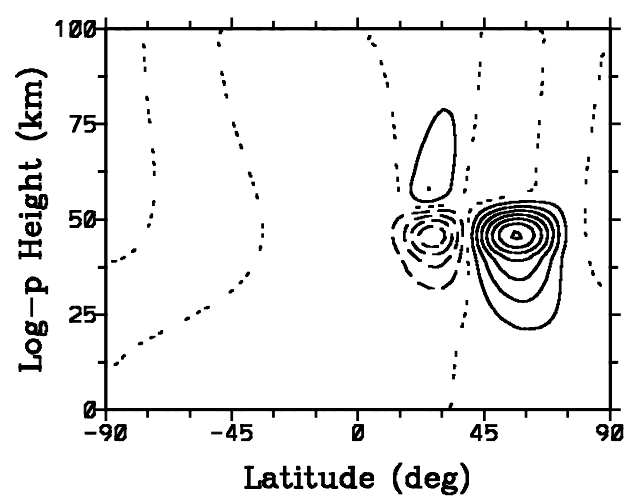

(b)

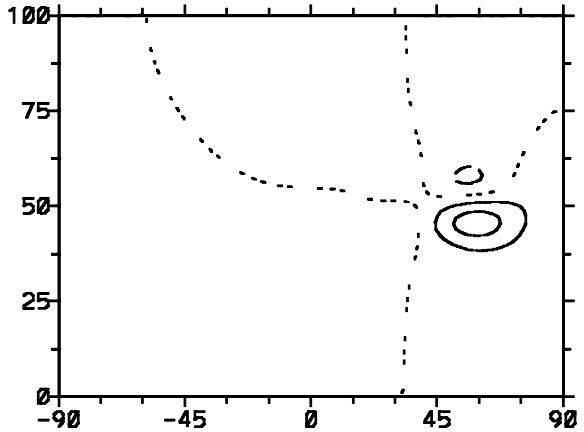

(d)

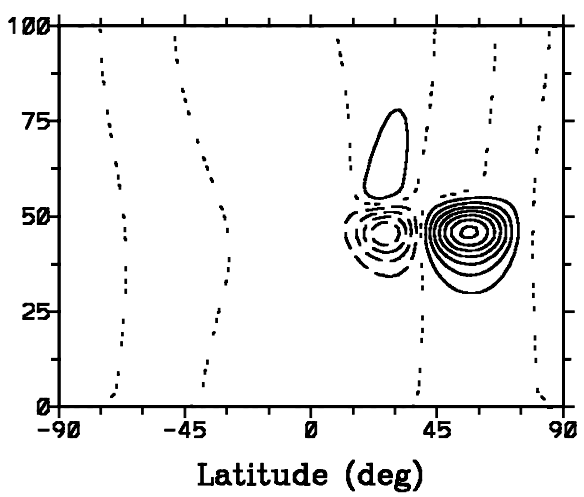

Figure 9. As Fig. 8 but for temperature. All contour intervals are $1 \mathrm{~K}$; dashed contours are negative.

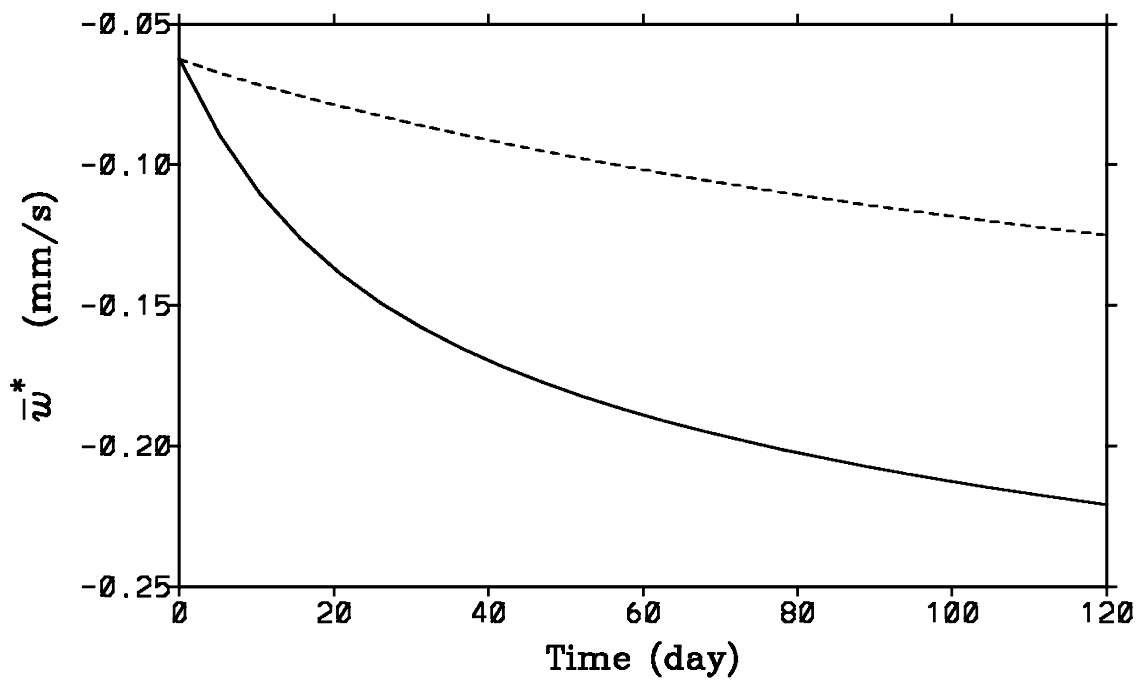

Figure 10. Time series of $\bar{w}^{*}$ on the $16 \mathrm{~km}$ level at $\phi=45^{\circ}$ from a run like that of Fig. 8 but with the forcing located $20 \mathrm{~km}$ lower. The solid curve corresponds to the uniform $\alpha=(1 / 6)$ day $^{-1}$ case and the dashed curve to the $\alpha(z)$ case. 
(a)

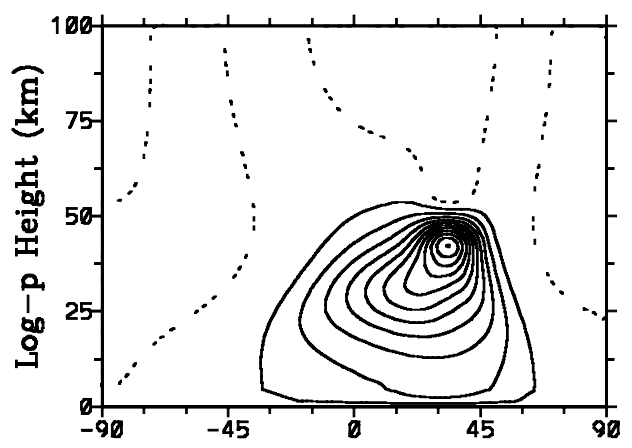

(c)

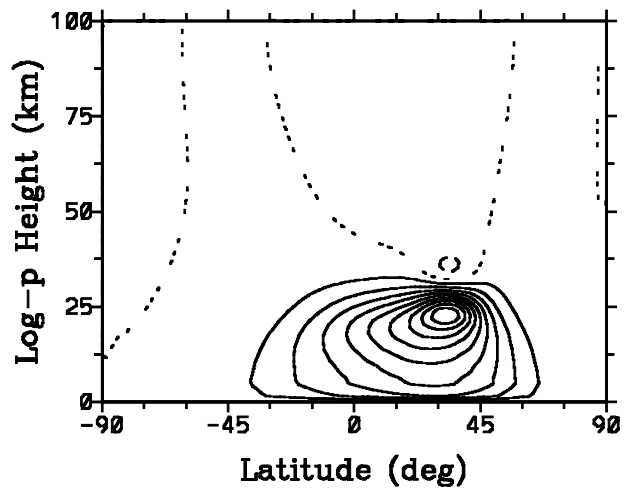

(b)

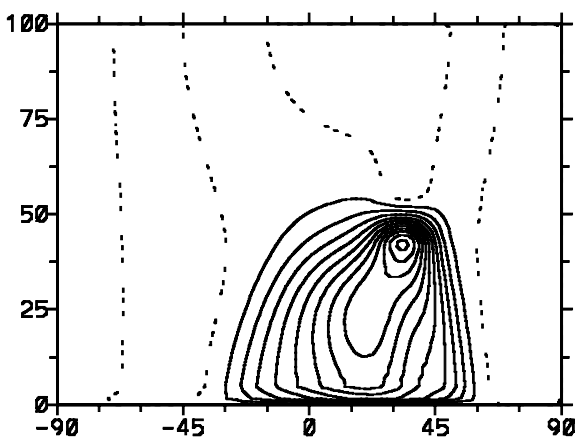

(d)

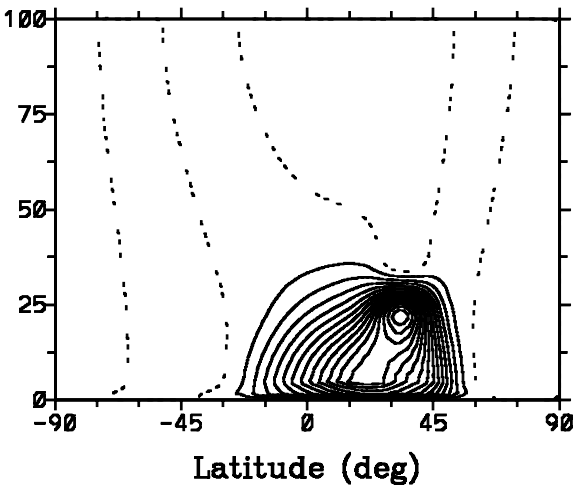

Figure 11. Contour plots of the $\cos (\omega t)$ component of the stream function produced by annually periodic forcing: (a) and (c) are for the $\alpha(z)$ case, (b) and (d) correspond to a uniform $\alpha=(1 / 6)$ day $^{-1}$. The force distribution parameters are $\phi_{1}=20^{\circ}, \phi_{2}=50^{\circ}$; for (a) and (b) $z_{1}=40 \mathrm{~km}$, for (c) and (d) $z_{1}=20 \mathrm{~km}$, with $z_{2}=z_{1}+20 \mathrm{~km}$. Contour intervals are $0.4 \mathrm{~kg} \mathrm{~m}^{-1} \mathrm{~s}^{-1}$ for (a) and (b), and $5 \mathrm{~kg} \mathrm{~m}^{-1} \mathrm{~s}^{-1}$ for (c) and (d); intermittent-dotted contours are zero. See text for further details.

Thus, unlike in the case of time-independent $\alpha$, the temperature change $\Delta \bar{T}$ is not constrained to overlap the diabatic-forcing region in the annual mean. For the case of an imposed forcing, (26) holds with $\delta \bar{T}_{\text {rad }}=0$ in regions where $\left\langle\bar{w}^{*}\right\rangle=0$. One such region is that above the forcing where it is now possible that $\langle\Delta \bar{T}\rangle \neq 0$, unlike in the downward control case.

We consider first the response of the model forced with an annually periodic heating $\delta \bar{T}_{\text {rad }}$ when $\alpha$ varies with time, and the difference with the constant $\alpha$ case. The time dependence of $\alpha$ is now given by

$$
\alpha(t)=\frac{1}{10 \text { day }}\left\{\frac{2}{3}-\frac{1}{3} \cos \left(\frac{2 \pi t}{365}\right)\right\} .
$$

As before, this is an idealization based on the annual cycle of $\alpha$ inferred from the radiative transfer scheme of Fomichev and Blanchet (1995) for the region of interest (high latitudes at about $20 \mathrm{~km}$, which is lower than that considered for Fig. 1(c)). An effect of time-dependent $\alpha$ is to change the rate of evolution to steady state at different times of the year. As a result, the transient induced during the late-winter and early-spring heating will decay faster during the summer season than if $\alpha$ were time 
(a)

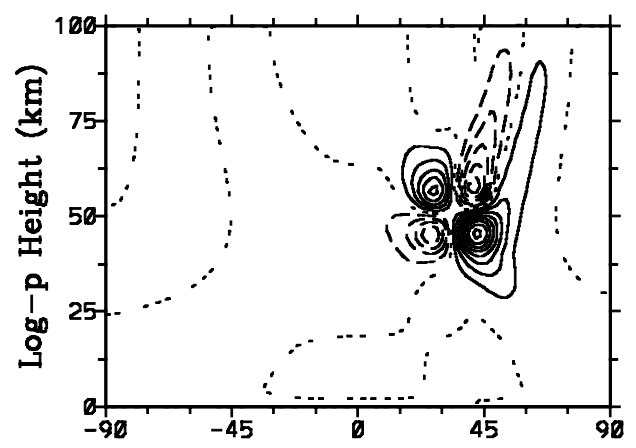

(c)

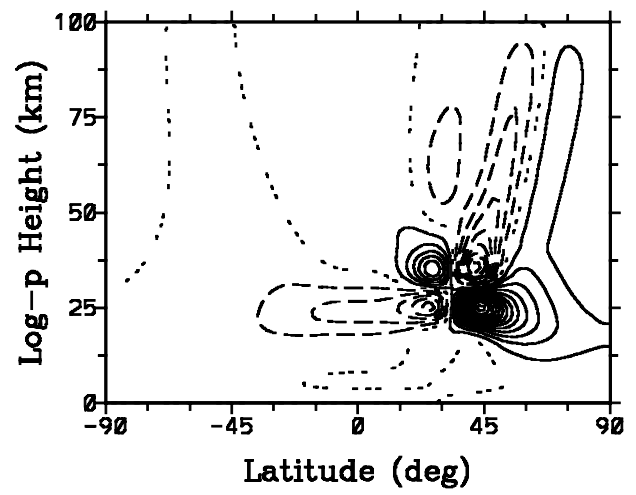

(b)

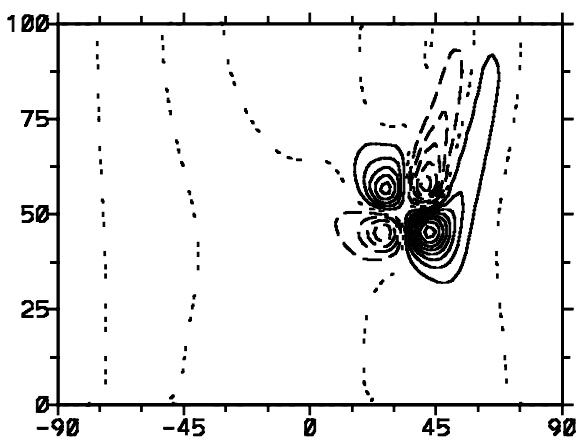

(d)

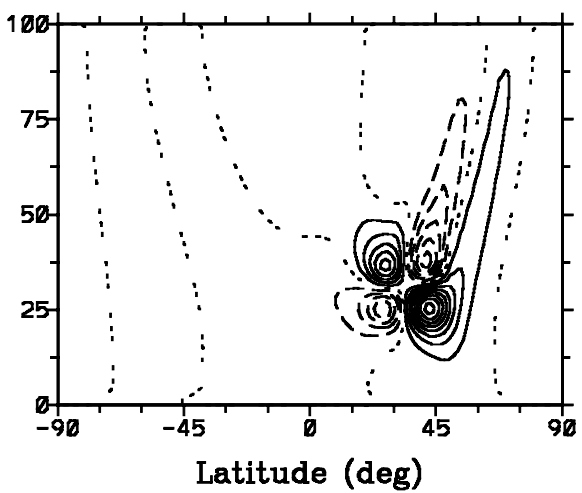

Figure 12. As Fig. 11 but for temperature. All contour intervals are $1 \mathrm{~K}$; dashed contours are negative.

independent. During the winter season the weaker radiative damping rate results in a larger temperature response but a weaker diabatic circulation. This is shown in Fig. 14 in a time series of $\Delta \bar{T}$ at a point in the heating region. Thus, $\alpha=\alpha(t)$ introduces a seasonal asymmetry in the evolution compared to the time-independent $\alpha$ case. This difference can lead to a transient signature in $\langle\Delta \bar{T}\rangle$, with warming located above the region of cooling. For the diabatic heating considered here, however, this signature is negligible with a magnitude of about $1 \%$ of $\langle\Delta \bar{T}\rangle$ in the cold region (Fig. 15). This can partly be attributed to the weakness of the transience during the seasonal cycle: the region of warming above the region of cooling has a peak amplitude less than $10 \%$ of the latter during most of the heating period. To the extent that this example mimics late-winter/spring polar ozone loss, it appears that the warming that is observed to occur above the cooling (Ramaswamy et al. 1996) cannot be accounted for by this mechanism.

The value of $\alpha$ used in the time-independent case was $\alpha\left(t_{2}\right)=(1 / 12)$ day $^{-1}$ where $t_{2}=4$ months is the time of peak diabatic forcing. This choice is arbitrary but makes the comparison more straightforward since the magnitude of the temperature (and zonal wind) response is determined by the typical values of $\alpha$ when the forcing is engaged.

A similar experiment to the one above was performed using a forcing with a time dependence roughly resembling that of stratospheric planetary-wave drag. In this case 


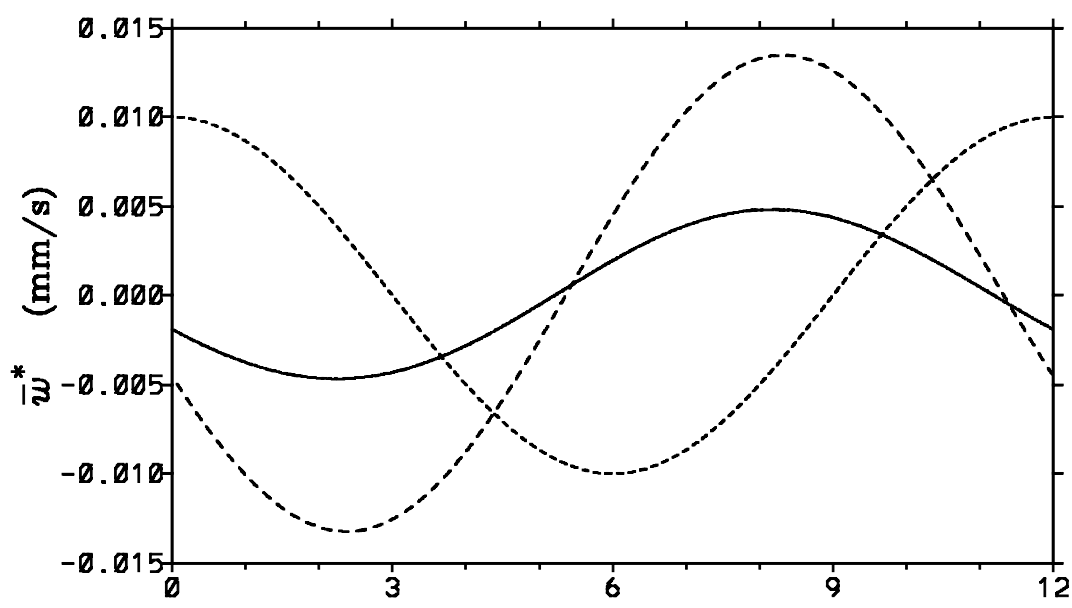

(a)

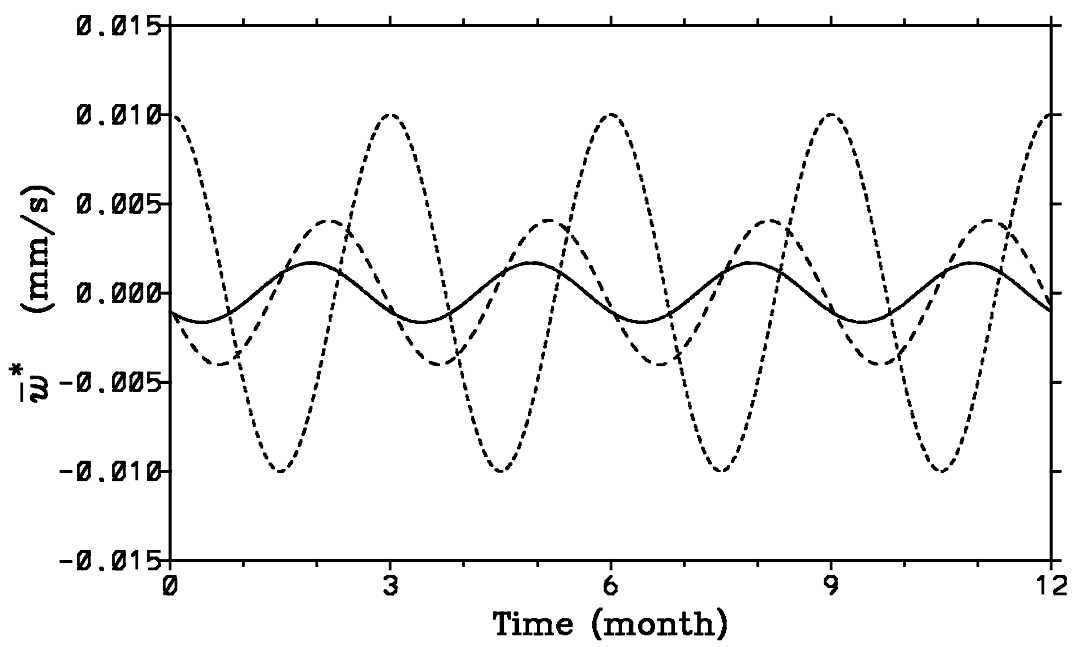

(b)

Figure 13. Time series of $\bar{w}^{*}$ on the $16 \mathrm{~km}$ surface at $42^{\circ}$ for uniform $\alpha=(1 / 6) \mathrm{day}^{-1}$ (long-dashed curves) and for $\alpha(z)$ (solid lines) for $\omega$ corresponding to: (a) a period of 1 year, and (b) a period of $(1 / 4)$ year. The time dependence of the forcing (short-dashed curves) is represented with an arbitrary amplitude. The force distribution parameters are $\phi_{1}=20^{\circ}, \phi_{2}=50^{\circ}, z_{1}=40 \mathrm{~km}$ and $z_{2}=60 \mathrm{~km}$. See text for further details.

the time dependence of $\alpha$ is given by

$$
\alpha(t)=\frac{1}{8.1 \text { day }}\left\{1-0.35 \cos \left(\frac{2 \pi t}{365}\right)\right\} .
$$

For the reference constant $\alpha$ case the minimum value of $\alpha(t)$, which is $(1 / 12.5)$ day $^{-1}$, was used since it corresponded to the time of peak forcing. It is evident that the increased damping during the summer season diminishes the transient tail of the winter season forcing (Fig. 16). This translates into a transient pattern in $\langle\Delta \bar{T}\rangle$ (Fig. 17), with a response above the forcing, even as the system reaches a steady oscillatory state. The larger $\langle\Delta \bar{T}\rangle$ amplitude in the constant $\alpha$ case is due to the fact that the damping rate is weaker than in the $\alpha(t)$ case except at a single point in time. This highlights the fact that the $\langle\Delta \bar{T}\rangle$ pattern in the time-dependent $\alpha$ case is not an artefact of start-up transience related to the finite duration of the model simulation. If that were the case, 


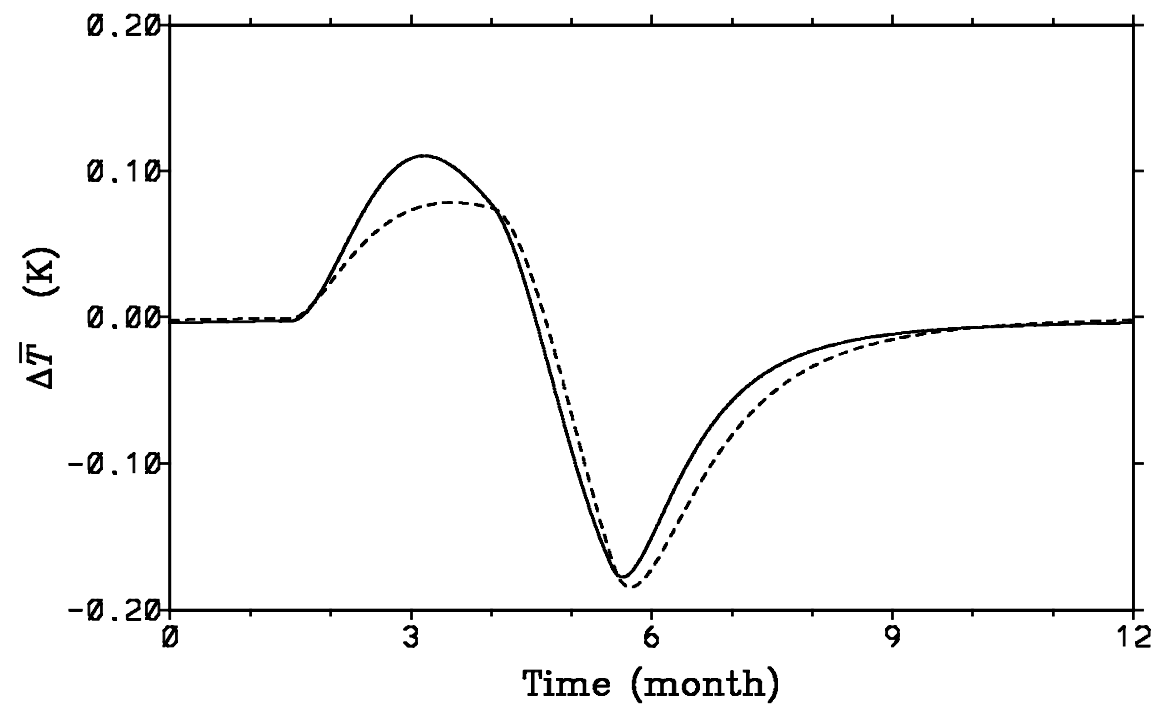

Figure 14. Time series of $\delta \bar{T}_{\text {rad }}$ induced $\Delta \bar{T}$ at $25 \mathrm{~km}$ and $76^{\circ}$ for the $\alpha(t)$ case (solid line) and constant $\alpha=(1 / 12)$ day $^{-1}$ case (dashed line). See text for details.

then start-up transience would also appear in the uniform $\alpha$ case, which has a weaker radiative damping rate.

\section{DISCUSSION AND CONCLUSION}

We have investigated the zonal-mean response of the quasi-geostrophic TEM system to an imposed switch-on or periodic forcing, given prescribed radiative damping profiles $\alpha(\phi), \alpha(z)$ and $\alpha(t)$. Spatial variation of radiative damping introduces spatial dependence in the rate of evolution to steady state; temporal variation of radiative damping leads to the rate of evolution towards steady state being time dependent. For oscillatory forcing, compared to the uniform $\alpha$ case the lag between $\bar{w}^{*}$ and $\bar{T}$ is no longer the same at every point in the meridional plane. If $\alpha$ varies only in space then the lag depends on the local value of $\alpha$. Time-dependent $\alpha$ introduces a temporal modulation of the solution amplitude which further affects the lag. The only other factor which introduces a spatial variation in the rate of evolution to steady state is the Coriolis parameter (the time taken to reach steady state increases as the Coriolis parameter decreases at low latitudes).

The spatial distribution of solutions is affected by the local value of the radiative damping rate. Regions with weaker $\alpha$ have reduced latitudinal confinement of streamlines and less vertical streamline penetration below the forcing level. This means a weaker $\bar{w}^{*}$ response but generally a greater induced $\bar{T}$. In the oscillatory case the solutions are trapped in a transient pattern in such regions. The solutions of the system with oscillatory forcing reflect the behaviour of the radiative damping rate, provided that the frequency of the forcing is not too high-in the latter case the system is in the transient limit and not sensitive to $\alpha$. But for the middle atmosphere we are generally interested in cases where the forcing period is longer than the radiative damping time-scale.

For the missing gravity-wave-drag problem of Garcia and Boville (1994), the important aspect is the sensitivity of the temperatures inside the polar vortex, on account of the longer radiative damping time-scale there. This is evident in both the steady-state and annually periodic forcing experiments of section 3 . Even if the polar circulation 


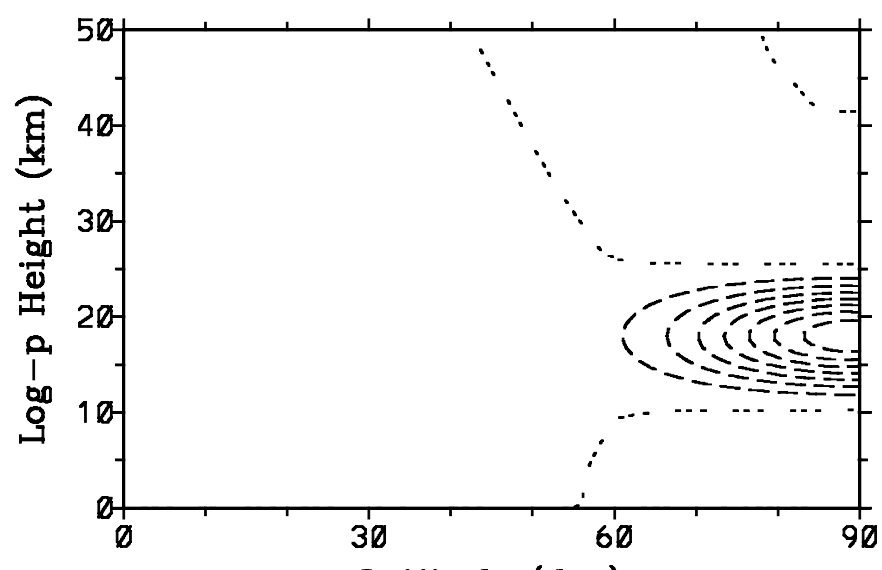

(a)

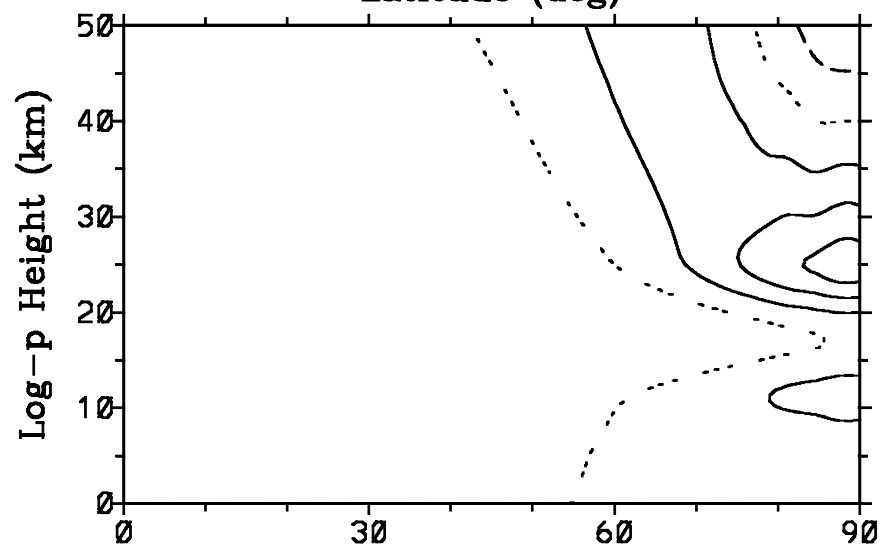

(b)

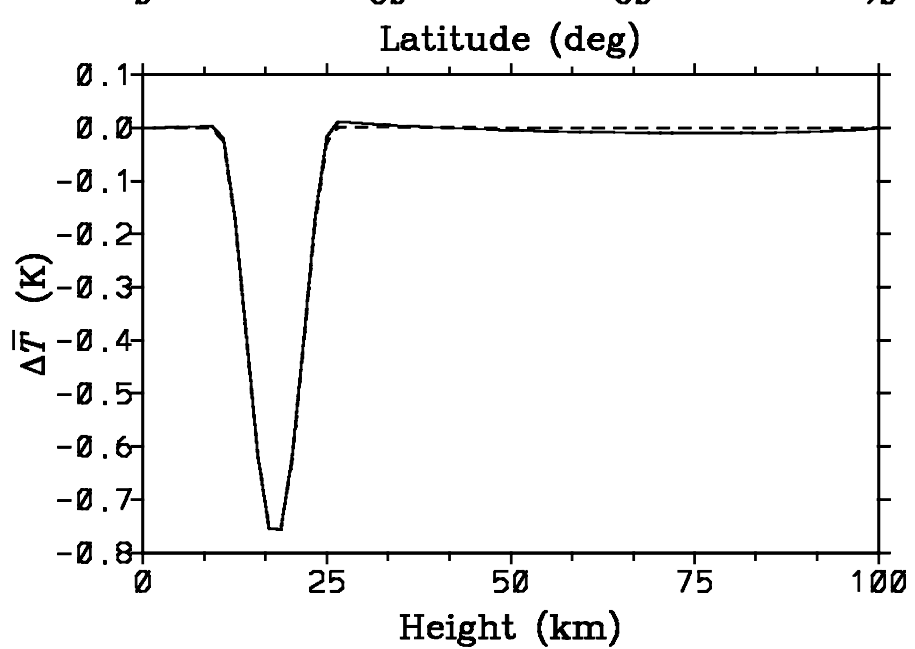

(c)

Figure 15. Annual mean $\Delta \bar{T}$ for: (a) $\alpha(t)$, and (b) the difference from the constant $\alpha$ case for imposed polar cooling (mimicking ozone depletion) corresponding to Fig. 14. The contour interval is $0.1 \mathrm{~K}$ in (a) and $0.003 \mathrm{~K}$ in (b); intermittent-dotted contours are zero and dashed contours are negative. (c) Gives the vertical profile of the annual mean $\Delta \bar{T}$ at $90^{\circ}$ for the $\alpha(t)$ case (solid line) and the constant $\alpha$ case (dashed line). See text for details. 


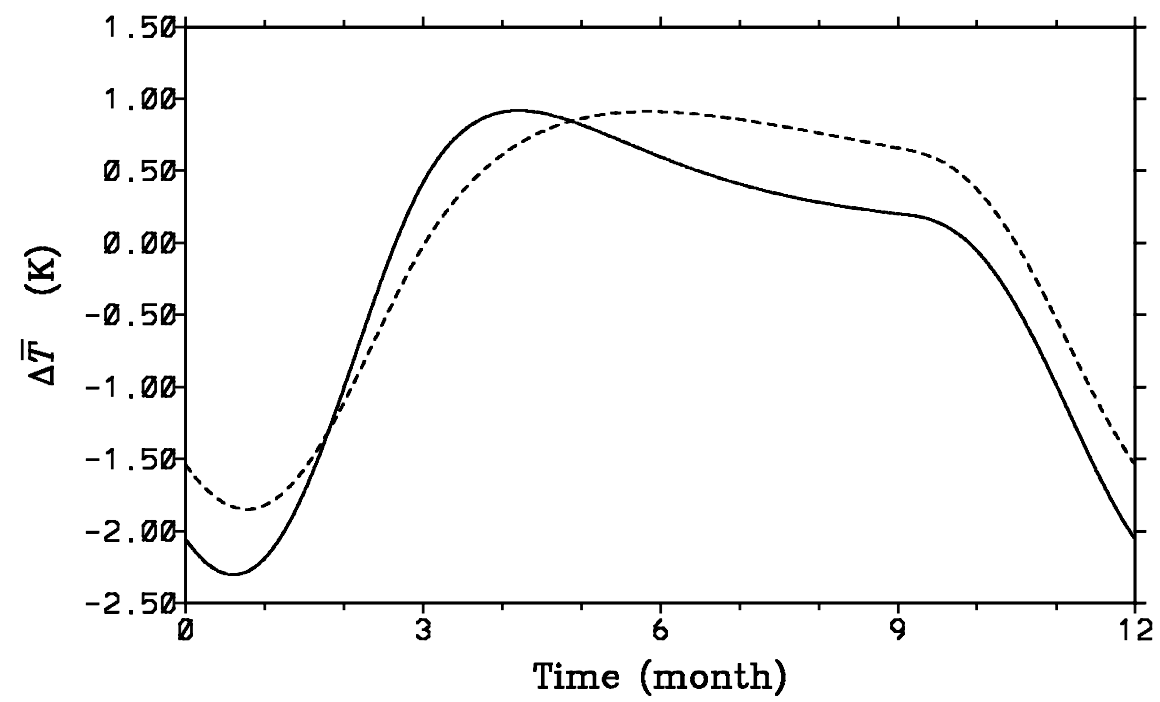

Figure 16. Time series of $\mathscr{\mathcal { F }}$ induced $\Delta \bar{T}$ at $39 \mathrm{~km}$ and $65^{\circ}$ for the $\alpha(t)$ case (solid line) and for the constant $\alpha$ case (dashed line). See text for details.

is weaker than is the case with a stronger damping rate, the temperature can be driven further from radiative equilibrium. On the basis of the $\alpha(z)$ experiments (e.g. Figs. 8 and 9) it can be inferred that the temperatures in the polar vortex interior in the lower stratosphere are sensitive to high-latitude drag in the mesosphere, in agreement with the results of Garcia and Boville (1994). The fact that the system is in a more transient regime in regions with weaker radiative damping rates appears to be more than offset by the increased temperature response. In the case of sub-polar-wave drag in the stratosphere (e.g. Figs. 2, 3, 4 and 5), reduced radiative damping over the pole leads to a larger-amplitude temperature response extending closer to the pole. This suggests that wave drag outside the polar vortex in the lower stratosphere has to be considered in addition to mesospheric gravity-wave drag in attempting to explain the temperatures inside the southern hemisphere polar vortex.

The results presented here pertaining to the mass flux through the $16 \mathrm{~km}(100 \mathrm{hPa})$ surface indicate that for the annually periodic case the maximum downwelling cannot be regarded as in phase with the forcing. There is a lag between the peak forcing and peak downwelling at midlatitudes (Fig. 13) which depends primarily on the altitude of the forcing. The lag increases as the forcing is moved to higher altitudes. Variation of the radiative damping rate with altitude has a secondary effect for annually periodic forcing. In particular, the weaker radiative damping rates in the lower stratosphere increase the lag between peak forcing and peak downwelling by less than a week for the idealized experiments conducted here.

It must be noted that the details of the lag apply for the specific force distribution used here. The force distribution inferred from observations is latitudinally broader, which according to the results of Haynes et al. (1991) should translate into a more steady-state character of the circulation and therefore a reduced lag. The observed force distribution is also deeper and extends to $16 \mathrm{~km}$, which implies that the downwelling behaviour at $16 \mathrm{~km}$ is the net effect of the contributions from different levels. The implication of the experiments conducted here for the conclusions of Appenzeller et al. (1996) is, nevertheless, that the delay between the peak wave drag in the 'overworld' 


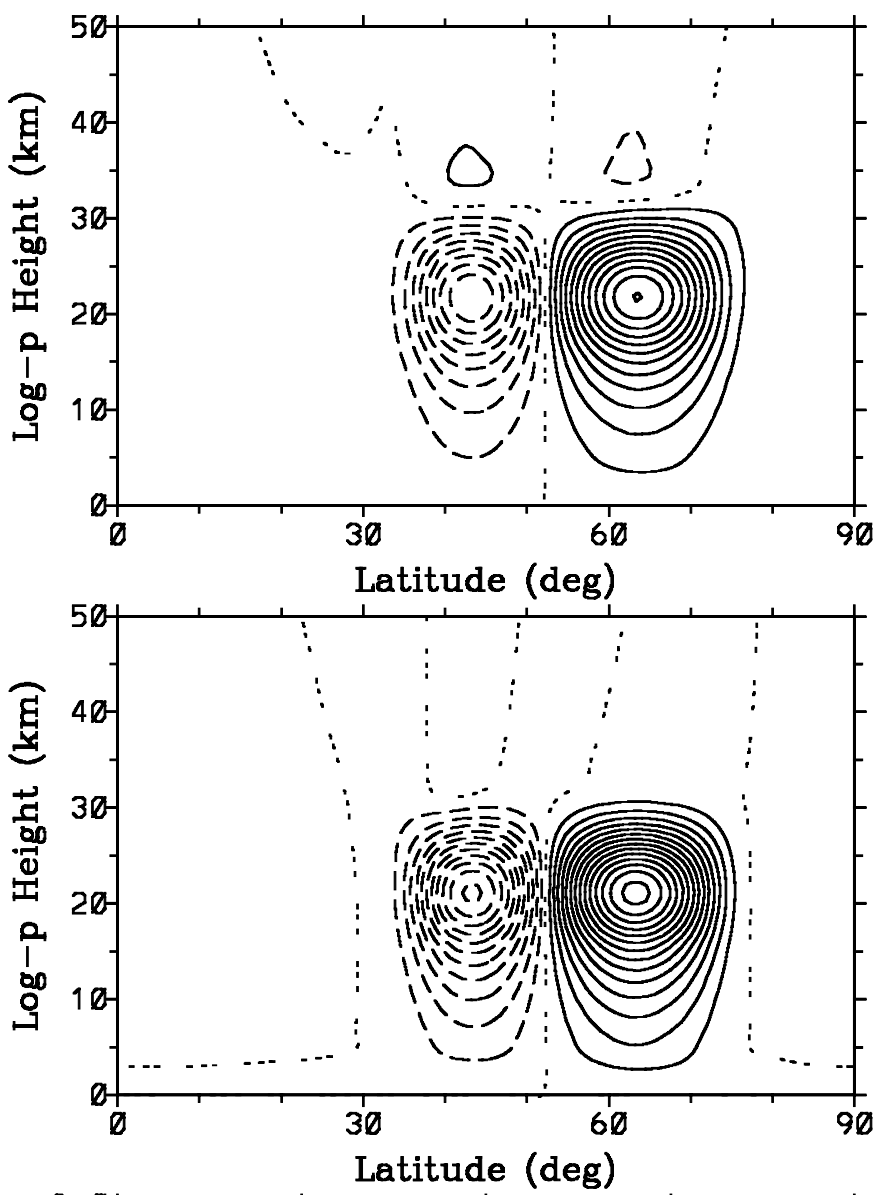

(a)

(b)

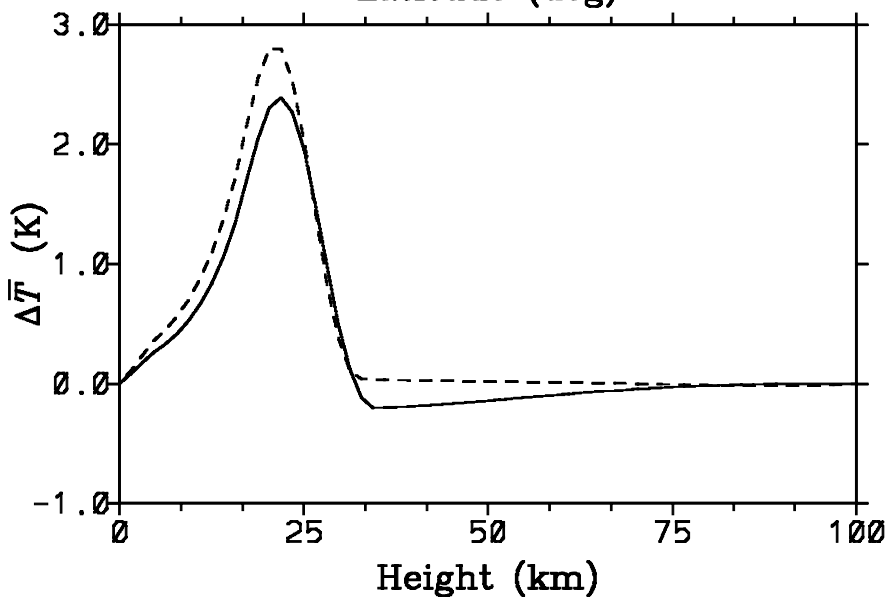

(c)

Figure 17. Annual mean $\Delta \bar{T}$ for: (a) $\alpha(t)$, and (b) constant $\alpha$, for the case of an imposed force corresponding to Fig. 16. Contour intervals are $0.2 \mathrm{~K}$; intermittent-dotted contours are zero and dashed contours are negative. (c) Gives the vertical profile of the annual mean $\Delta \bar{T}$ at $65^{\circ}$ for (a) (solid line) and (b) (dashed line). See text for details. 
(a)

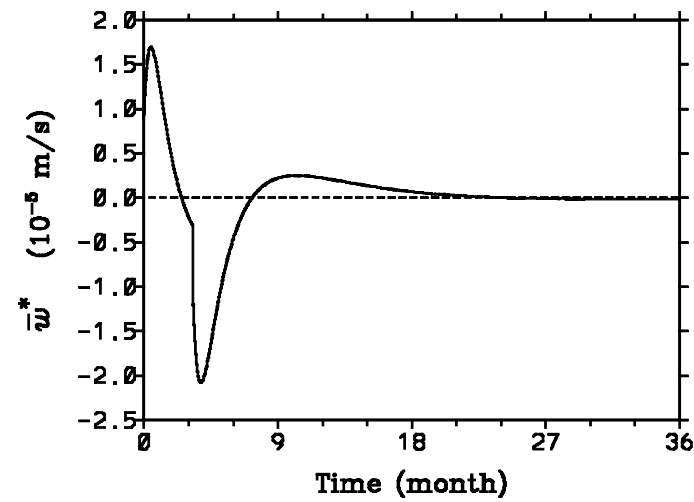

(b)

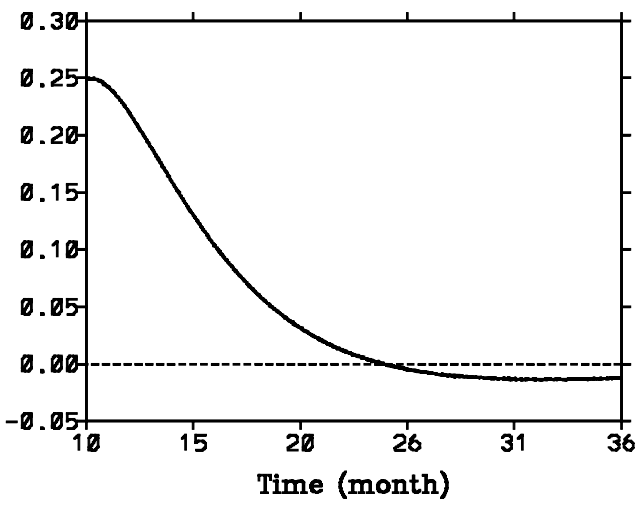

Figure 18. (a) Time series of $\bar{w}^{*}$ at $39 \mathrm{~km}$ and $-14^{\circ}$ for 36 model months. The force is active only during the first 100 days with a uniform amplitude of $-2.5 \mathrm{~m} \mathrm{~s}^{-1}$ day ${ }^{-1}$ and distribution specified by $\phi_{1}=35^{\circ}, \phi_{2}=65^{\circ}$, $z_{1}=30 \mathrm{~km}$ and $z_{2}=50 \mathrm{~km}$. The radiative damping rate has a constant value of $(1 / 6)$ day $^{-1}$. (b) Shows an enlarged section of the same time series starting at 10 model months. See text for further details.

(above $16 \mathrm{~km}$ ) and the mass outflow into the troposphere from the lowermost stratosphere is not all due to seasonal evolution of tropopause height.

Time variations in the radiative damping rate do not prevent annual mean downward control from being established, but the condition $\left\langle\bar{w}^{*}\right\rangle=0$ now no longer implies that $\langle\Delta \bar{T}\rangle=\left\langle\delta \bar{T}_{\mathrm{rad}}\right\rangle$. This applies for diabatic forcing, and outside the downward control region for mechanical forcing. An example intended to mimic the polar ozone hole showed a net warming above the region of ozone-induced cooling, but its magnitude was much too small to account for the observed warming. This reflects the weakness of the transient warming that accompanies the evolution of the diabatic forcing produced by ozone loss.

There is a significant degree of interannual variability in the middle atmosphere, linked to external forcing (mechanical or diabatic) as well as internal modes. In the zonal mean both are folded into the EP flux divergence and radiative equilibrium temperature variations on a mean seasonal cycle. Phenomena such as the ozone hole have not yet equilibrated, and seasonal ozone loss has been increasing in magnitude over much of the last 20 years (WMO 1999). Wave drag (Rossby-wave and gravity-wave breaking and thermal dissipation) also exhibits interannual variability in its distribution (e.g. sudden warmings, Quasi-Biennial Oscillation (QBO) link to polar vortex intensity (Holton and Tan 1980)). There is a start-up transient which persists for the first period of a steady oscillatory forcing when starting the system from rest (not shown). So a forcing with annual periodicity and interannually varying amplitude can be expected to maintain a transient component on top of the equilibrated mean.

The persistence of transients is somewhat counter-intuitive since the radiative damping time-scale is much shorter than a year even at lower altitudes and in the polar night. The reason for their long life is the diabatic circulation, which acts to divert part of the energy input by the forcing (mechanical or diabatic) into a non-local thermal reservoir by driving the temperature away from radiative equilibrium (Snieder and Fels 1988). When the forcing is switched off, some of the stored thermal energy gets converted into a meridional circulation which redistributes this energy. This circulation acts to produce adiabatic cooling or warming. As a result, the temperature does not decay exponentially with an e-folding rate of $\alpha$, but at a much slower rate. In the absence of the diabatic 
circulation the problem would, in contrast, be completely local and exhibit exponential decay. The zonal wind is damped through the temperature to which it is coupled via thermal-wind balance. Consequently, it evolves over similar time-scales.

The non-locality (ellipticity) which is associated with the diabatic circulation leads to a complicated pattern of evolution. Even though no homogeneous (unforced) dynamics are possible in the balanced TEM system, there is an oscillatory type of behaviour that results from the coupling of non-locality with relaxational radiative damping. Regions of forcing (mechanical or radiative) induce heating in adjacent regions. This leads to a decaying pattern of heating and circulation propagation which is particularly apparent after a forcing of finite duration is switched off. In Fig. 18 a time series of $\bar{w}^{*}$ is shown which demonstrates the non-monotonic behaviour after the forcing is switched off: there is an oscillation with a rapidly decaying amplitude which persists for several years.

\section{ACKNOWLEDGEMENTS}

This work represents part of KS's PhD thesis at the University of Toronto. He wishes to acknowledge support from an Ontario Graduate Scholarship, the Canadian MAM project and the university. TGS is supported by the Natural Sciences and Engineering Research Council and the Meteorological Service of Canada.

Andrews, D. G., Holton, J. R. and Leovy, C. B.

Appenzeller, C., Holton, J. R. and Rosenlof, K. H.

Fomichev, V. I. and Blanchet, J.-P.

Garcia, R. R.

Garcia, R. R. and Boville, B. A.

Haynes, P. H., Marks, C. J., McIntyre, M. E., Shepherd, T. G. and Shine, K. P

Holton, J. R. and Tan, H.-C.

Holton, J. R., Haynes, P. H., McIntyre, M. E., Douglass, A. R., Rood, R. B. and Pfister, L.

Ramaswamy, V., Schwarzkopf, M. D. and Randel, W. R.

Rosenlof, K. H. and Holton, J. R.

Sankey, D.

Snieder, R. K. and Fels, S. B.

WMO

\section{REFERENCES}

1987

1996

1995

1987

1994

1991

1980

1995

1996

1993

1998

1988

1999
Middle atmosphere dynamics. International Geophysics Series, Vol. 40. Academic Press, Orlando, USA

Seasonal variation of mass transport across the tropopause. J. Geophys. Res., 101, 15071-15078

Development of the new CCC/GCM longwave radiation model for extension into the middle atmosphere. Atmos.-Ocean, 33, 513-529

On the mean meridional circulation of the middle atmosphere. J. Atmos. Sci., 44, 3599-3609

'Downward control' of the mean meridional circulation and temperature distribution of the polar winter stratosphere. J. Atmos. Sci., 51, 2238-2245

On the 'downward control' of extratropical diabatic circulations by eddy-induced mean zonal forces. J. Atmos. Sci., 48, 651678

The influence of the equatorial quasi-biennial oscillation on the global circulation at $50 \mathrm{mb}$. J. Atmos. Sci., 37, 2200-2208

Stratosphere-troposphere exchange. Rev. Geophys., 33, 403-439

Fingerprint of ozone depletion in the spatial and temporal pattern of recent lower-stratospheric cooling. Nature, 382, 616-618

Estimates of the stratospheric residual circulation using the downward control principle. J. Geophys. Res., 98, 10465-10479

'Dynamics of upwelling in the equatorial lower stratosphere'. $\mathrm{PhD}$ thesis, University of Cambridge

The flywheel effect in the middle atmosphere. J. Atmos. Sci., 45, 3996-4004

'Scientific assessment of ozone depletion' 1998. Global Ozone Research and Monitoring Project. Report No. 44, World Meteorological Organization, Geneva, Switzerland 